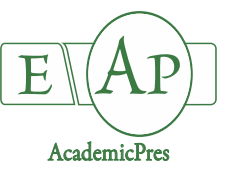

Shokouhian A and Omidi $\mathrm{H}$ (2021)

Notulae Botanicae Horti Agrobotanici Cluj-Napoca

Volume 49, Issue 3, Article number 12063

DOI: $10.15835 /$ nbha 49312063

Research Article

\title{
Sugar beet (Beta vulgaris L.) germination indices and physiological properties affected by priming and genotype under salinity stress
}

\author{
Ali SHOKOUHIAN ${ }^{1}$, Heshmat OMIDI ${ }^{2 *}$ \\ 'Shahed University, Department of Agronomy, Tehran, Iran;_alishokouhian@gmail.com \\ ${ }^{2}$ Shahed University, Agricultural College and Medicinal Plant Research Center, Tehran, \\ Iran; omidi@shahed.ac.ir ( ${ }^{*}$ correspondingauthor)
}

\begin{abstract}
Seed priming has proved to be an effective method in imparting stress tolerance to plants using natural and/or synthetic compounds to treat the seeds before germination. The present study was designed to investigate the physiological mechanism of seed priming with $\mathrm{ZnSO}_{4}$ (osmopriming) and distilled water (hydropriming) on sugar beet genotypes ('Shokofa', 'Sina', 'Paya', 'Turbata', and 'Aria') germination indices, seedling growth parameters, and biochemical properties under salinity stress $(0,2,5$, and $12 \mathrm{dS} / \mathrm{m} \mathrm{NaCl})$. A significant reduction in germination percentage (33.23\%), germination rate (77.2\%), chlorophyll a, b, and total contents (43.9, 31.9, and 39.9\%, respectively) while, a significant increase in radical, plumule, and seedling length (57.1, 44.4, and 51.2\%, respectively), seedling vigour index (48.9\%), superoxide dismutase activity (61.3\%), proline (54.0\%) and sugar (56.3\%) contents were achieved at $12 \mathrm{dS} / \mathrm{m} \mathrm{NaCl}$ in compared to the control treatment. Seed hydropriming and osmopriming caused significant improvements in photosynthetic pigments, antioxidant enzyme activity, and proline content reflected in high germination percentage and rate as well as seedling vigour index and reduced mean germination time under salinity. 'Paya' and 'Aria' genotypes had a superiority according to the germination percentage and seedling vigour index, respectively. The hydropriming of 'Paya' genotype resulted in the highest germination percentage (95\%) under high level of salinity $(12 \mathrm{dS} / \mathrm{m})$ which $11.84 \%$ increase compared to the control treatment. Hydropriming of 'Sina' seeds showed the highest chlorophyll a and total, and carotenoids under non-stress conditions (22.89, 31.65, and $2116.6 \mu \mathrm{g} / \mathrm{g} \mathrm{FW}$ ). Also, hydropriming by increases chlorophyll b content led to the modulation of the negative effects of high salinity stress $(12 \mathrm{dS} / \mathrm{m})$. In conclusion, different seed priming treatments in sugar beet seeds improved the salinity tolerance by physiological characteristics nonetheless hydropriming was the most effective treatment to get higher germination indices in 'Paya' and 'Aria' genotypes.
\end{abstract}

Keywords: hyrdopriming; osmopriming; photosynthetic pigments; proline content; salinity tolerance

Abbreviations: GP, germination percentage; GR, germination rate; RL, radical length; PL, plumule length; SL, seedling length; SVI, seedling vigor index; Chl, chlorophyll; ROS, reactive oxygen species; SOD, superoxide dismutase

Received: 02 Sep 2020. Received in revised form: 16 Aug 2021. Accepted: 23 Aug 2021. Published online: 17 Sep 2021.

From Volume 49, Issue 1, 2021, Notulae Botanicae Horti Agrobotanici Cluj-Napoca journal uses article numbers in place of the traditional method of continuous pagination through the volume. The journal will continue to appear quarterly, as before, with four annual numbers. 


\section{Introduction}

Sugar beet (Beta vulgaris L.) is one of the most recently domesticated crops, belongs to the order Caryophyllales (Skorupa et al., 2019). It is an important root crop in the world for sugar production, where its taproots are used. Sugar beet is the second most important source of sugar worldwide, after sugar cane, providing annually million tonnes of sugar for consumption and beet pulp for animal feed. The world production of sugar from sugar beet in 2018 was approximately 42 million metric tons, accounting for nearly $30 \%$ of the world's sugar supply (Lv et al., 2019). Sugar beet pulp is especially rich in polysaccharides, such as cellulose (20-24\%), hemicellulose (25-36\%), and pectic substances (15-25\%).

Among abiotic stresses, high concentrations of salt in the soil can result in severe detrimental factors, such as poor germination, seedling establishment, and crop yield (Carvalho et al., 2011). Salinity is an increasing problem affecting crop productivity in many irrigated arid and semi-arid areas of the world. Plant growth is affected, and detrimental effects include (i) reduced water availability, due to an osmotic effect from high concentrations of soluble salts in the root medium, (ii) ion toxicity, as a result of the accumulation of $\mathrm{Na}^{+}$and $\mathrm{Cl}$, (iii) oxidative stress, resulting from an overproduction of reactive oxygen species (ROS) and (iv) acute $\mathrm{K}^{+}$ deficiency as a result of massive $\mathrm{K}^{+}$leak from depolarized cells (Moreno et al., 2018). In general, the adaptive responses of plants to salt stress can be grouped into three categories: osmotic stress tolerance, ion exclusion, and tissue tolerance to salinity (Yang and Guo, 2018). Feghhenabi et al. (2020) reported that the response to salinity stress at each stage of growth varies not only among plant species but also among genotypes or cultivars. Most crop species are sensitive to salinity stress at early growth stages, including the germination and seedling establishment stages. During germination and emergence, plant survival is likely the most vital indicator of salinity tolerance, whereas afterward yield and growth reduction may be considered as the final tolerance criterion (Saadat and Homaee, 2015).

Nowadays, seed priming has become common practice to increase the rate and uniformity of seed germination and emergence, and many patented seed priming treatments targeting horticultural species (tomato, pepper, onion, sugar beet, lettuce, and Brassica) are commercially available (Paparella et al., 2015). Seed priming is an efficient method for increasing seedling vigour index (SVI) and synchronization of germination, as well as the growth of seedlings of many crops under stressful conditions (Aghighi Shahverdi et al., 2017). In this process, one interesting approach to the question of the ameliorative effects of priming solutions on stress tolerance is that seed priming with nutrients or hormones can also result in alleviated oxidative stress (Subramanyam et al., 2019). Seed priming as a pre-sowing technique can improve radicle emergence, germination percentage (GP), germination rate (GR), SVI, seedling establishment, and yield by making changes in metabolic activities in the seeds of many crops (Mosavikia et al., 2020). The effectiveness of seed priming depends on the plant species, the type and concentration of priming solution, the priming duration, and temperature and storage conditions (Aghighi Shahverdi et al., 2017; Khaing et al., 2020). During pre-germination metabolic activities, structural (membrane protection during imbibition) and genetic repair, RNA and protein synthesis and antioxidant mechanism take place in primed seed, which ensures its proper germination and seedling development (Saddiq et al., 2019). Hydropriming is the simplest approach to hydrate seeds and minimize the use of chemicals. It reported that hydropriming treatments increased the GP, GR, radical length (RL), as well as plumule, radical, and seedling dry weight (Toklu, 2015). The results of Anwar et al. (2020) suggested that seed priming with $\mathrm{GA}_{3}$ and $\mathrm{KNO}_{3}$ synergistically promoted the photosynthetic pigments and nutrients uptake in cucumber seedlings, thus leading to improve plant growth.

Zinc is an important microelement essential for plants and humans. It is the cofactor of many enzymes like DNA, RNA polymerases, and zinc finger proteins (Rehman et al., 2018). Its deficiency in agricultural soils is common all over the developed and developing countries especially in drought and salt areas (Mahmood et al., 2019). Besides, priming seeds in zinc-containing solutions were shown to increase zinc content in the primed seeds and/or to contribute to a better seedling growth and yield. Pavia et al. (2019) reported that the priming improves germination and seed reserve utilization, growth, antioxidant responses, and membrane 
stability at early seedling stage of Saudi sorghum varieties under drought stress. Many studies have developed salt-tolerant lines or cultivars using conventional plant breeding, but seed priming is a simple, promising, and non-GMO technique to pre-condition stress tolerance in plants before germination. The objectives of this study were to (i) characterize the germination and physiological response to different salinity stress, hydropriming and osmopriming and (ii) investigate whether hydropriming and osmopriming induce tolerance to salinity in seeds of sugar beet genotypes.

\section{Materials and Methods}

\section{Plant material and treatments}

To determine the effect of different primes on germination indices and physiological traits of sugar beet genotypes, an experiment was conducted at Shahed University, Tehran, Iran, in 2019. Three factorial experiments were carried out to assess the effects of priming (unprimed as control, hydropriming, and osmopriming with zinc sulfate $\left.\left(\mathrm{ZnSO}_{4}\right)\right)$ on germination indices and physiological characteristics of five genotypes of sugar beet ('Shokofa', 'Sina', 'Paya', 'Turbata', and 'Aria') under salt stress (0, 2, 5, and 12 dS/m $\mathrm{NaCl}$ ) in a completely randomized design with three replications. Seeds of sugar beet genotypes were obtained from Sugar Beet Seed Institute, Karaj, Iran. Before experimenting, to determine of best priming duration was a pilot experiment, where five priming durations $(0,8,16,24$, and 32 hours) for each treatment (hydropriming and osmopriming) separately, were tested. The results showed that the best duration was 12 hours, that was used this data in the experiment. ISTA recommends germinating sugar beet seeds on between paper or top of the paper at constant or alternating temperatures, 25 or $20 / 30^{\circ} \mathrm{C}$, respectively. In the experiment, the seed of sugar beet was immersed in a $5 \%$ sodium hypochlorite solution for $10 \mathrm{~min}$ to ensure surface sterility. Then they were soaked in distilled water (as hydropriming) and $\mathrm{ZnSO}_{4}(0.5 \%)$ suspension for about 12 hours at $15^{\circ} \mathrm{C}$. Treated seeds were shade-dried for 12 hours. Zinc sulfate wad was purchased from Sigma-Aldrich, USA. Then the 50 seeds were placed in a Petri dish $(24 \times 1.5 \mathrm{~cm})$ with one piece of sterilized filter paper (Whatman No.1) and $12 \mathrm{ml}$ of distilled water (for control) or salinity solution based on various treatments was added to each Petri dish. Van't Hoff formula (1) was used prepared for salinity solution (Aghighi Shahverdi et al., 2017).

Formula 1) $\quad \Psi=$-MIRT

Whereas $\Psi$ : osmotic potential according to Bar; M: molarity; I: Van't Hoff factor $=2$ for $\mathrm{NaCl}$; R: constant number $=0.08206$ bar; $T$ : temperature according to Kelvin

Germinated seeds were counted on the second day was done daily and finally at the end of the testing period (14 days) was calculated GP, mean germination time (MGT), GR, germination uniformity (GU), and SVI according to the following formulas (Parmoon et al 2015; Aghighi Shahverdi et al 2017).
Germination percentage
$\mathrm{GP}=(\mathrm{N} \times 100) / \mathrm{M}$
Mean time of germination
$\left.\left.\mathrm{MTG}=\sum\right) \mathrm{Ni}\right) / \sum \mathrm{N}$
Germination rate
$\mathrm{GR}=\sum \mathrm{Ni} / \mathrm{Ti}$
Germination uniformity
$\mathrm{GU}=1 / \mathrm{N}^{*}$
Seed vigor index
$\mathrm{SVI}=\mathrm{GP} \times \operatorname{Mean}(\mathrm{SL})$

Whereas $\mathrm{N}=$ sum of germinated seeds at the end of the experiment, $\mathrm{M}=$ total planted seeds, $\mathrm{Ti}=$ number of days after germination, $\mathrm{N}^{*}=$ the number of days that germination reached 10 to $90 \%, \mathrm{SL}=$ Seedling length.

\section{Relative water content}

The relative water content of the leaves was determined using the methods of Shaw et al. (2002). Each disc (diameter $10 \mathrm{~mm}$ ) was placed into a separate glass-stoppered tube. Having ensured that there was no excess water on the discs, the fresh weight of each was recorded. The discs were then floated on $2 \mathrm{ml}$ of distilled water for $24 \mathrm{~h}$ in natural daylight. At the end of this period, the fully turgid samples were rapidly surface dried with 
filter paper and re-weighed. These discs were then dried at $60^{\circ} \mathrm{C}$ for $24 \mathrm{~h}$ and the dry weights established. The relative turgidity was calculated.

\section{Photosynthetic pigments}

For measuring the content of photosynthetic pigments was used Lichtenthaler and Buschmann (2001) method. According to this method, $0.25 \mathrm{~g}$ of fresh tissue was extracted using $5 \mathrm{ml} 80 \%$ acetone. The extract was centrifuged at $11000 \mathrm{rpm}$ for $10 \mathrm{~min}$. The optical density (O.D.) of the extract was measured at wavelengths $646.8,663.2$, and $470 \mathrm{~nm}$. The amount of pigment present in each sample was calculated according to the following equations (Shahverdi et al., 2019):

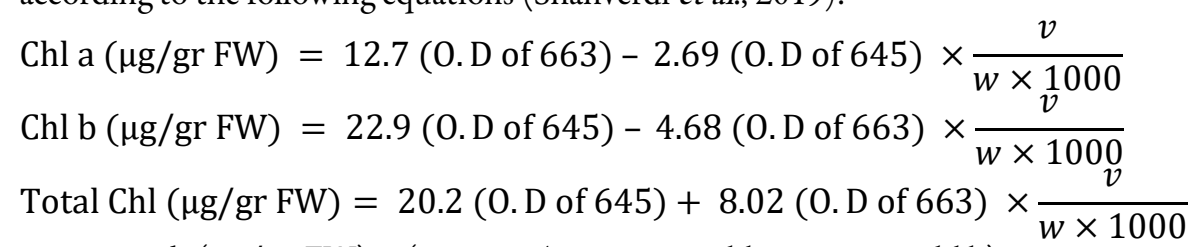

Carotenoids $(\mu \mathrm{g} / \mathrm{gr} \mathrm{FW})=(1000 \times \mathrm{A} 470-1.82 \times \mathrm{Chl} \mathrm{a}-85.02 \times \mathrm{Chl} \mathrm{b}) / 198$

Whereas W: the fresh weight by grams for extracted tissue; V: the final size of the extract in $80 \%$ acetone; O.D: optical density at a specific wavelength.

\section{Anthocyanin content}

After being thoroughly extracted in $3 \mathrm{ml}$ methanol- $\mathrm{HCl}(1 \% \mathrm{HCl}, \mathrm{v} / \mathrm{v})$, the samples were left at $4{ }^{\circ} \mathrm{C}$ in the refrigerator for 2 days. Later on, he extracts were filtered and the total anthocyanin content was measured by a UV-visible spectrophotometer as the difference between the absorbance at 530 and $657 \mathrm{~nm}$ wavelength and placed in the A530-A657 formula to eliminate the chlorophyll content in the extract, defined quantitatively as OD $530 \mathrm{~g}^{-1}$ fresh weight (Mancinelli, 1990).

\section{Protein and superoxide dismutase assay (SOD)}

Samples were frozen in liquid nitrogen and stored at $-30{ }^{\circ} \mathrm{C}$. One g of frozen sample was homogenized in a mortar with $5 \mathrm{ml}$ of $50 \mathrm{mM}$ potassium phosphate buffer $(\mathrm{pH} 7.5)$ containing $1 \mathrm{mM}$ ethylenediaminetetraacetic acid (EDTA), $1 \mathrm{mM}$ dithiothreitol and $2 \%$ polyvinyl pyrrolidone. The homogenate was centrifuged at 15,000 $\mathrm{g}$ for $25 \mathrm{~min}$ and the supernatant was used for protein and SOD assay. Protein percentage measurement according to the method of Bradford (1976). For SOD activity measurement, the method of Beauchamp and Fridovich (1971) was used which is briefly described here. About $3 \mathrm{ml}$ of the reaction mixture, containing $0.1 \mathrm{ml}$ of $200 \mathrm{mM}$ methionine, $0.01 \mathrm{ml}$ of $2.25 \mathrm{mM}$ nitro blue tetrazolium, 0.1 $\mathrm{ml}$ of $3 \mathrm{mM}$ EDTA, $1.5 \mathrm{ml}$ of $100 \mathrm{mM}$ potassium phosphate buffer, $1 \mathrm{ml}$ distilled water and $0.05 \mathrm{ml}$ of enzyme extraction, were taken in test tubes in duplicate from each enzyme sample. Two tubes without enzyme extract were taken as control. The reaction was started by adding $0.1 \mathrm{ml}$ riboflavin $(60 \mu \mathrm{M})$ and placing the tubes below a light source of two $15 \mathrm{~W}$ fluorescent lamps for $15 \mathrm{~min}$. The reaction was stopped by switching off the light and covering the tubes with black cloth. Absorbance was recorded at $560 \mathrm{~nm}$.

\section{Proline assay}

Proline was determined according to the method described by Bates et al. (1973). Approximately $0.5 \mathrm{~g}$ of fresh seedling was homogenized in $10 \mathrm{ml}$ of $3 \%$ aqueous sulfosalicylic acid. Then, this aqueous solution was filtered through Whatman's paper No. 2 and finally, $2 \mathrm{ml}$ of filtrated solution was mixed with $2 \mathrm{ml}$ acidninhydrin and $2 \mathrm{ml}$ of glacial acetic acid in a test tube. The mixture was placed in a water bath for $1 \mathrm{~h}$ at 100 ${ }^{\circ} \mathrm{C}$. The reaction mixture was extracted with $4 \mathrm{ml}$ toluene, cooled to room temperature, and the absorbance was measured at $520 \mathrm{~nm}$ with a spectrometer. 


\section{Sugar content}

Samples of fresh seedlings were weighed $(0.2 \mathrm{~g})$ and homogenized using $70 \%$ ethanol. Then they were filtered and pigments were removed by the use of benzene. An aliquot of $0.2 \mathrm{ml}$ of seedling extract was added to $1.0 \mathrm{ml}$ of $0.2 \%$ anthrone to react in a water bath for $10 \mathrm{~min}$ at $100{ }^{\circ} \mathrm{C}$. Soon after, the test tube 3 was cooled in an ice bath and then the absorbance was reared at $620 \mathrm{~nm}$, according to Yemm and Folkes (1953).

\section{Statistical analysis}

Analysis of variance (ANOVA) was carried out using Statistical Analysis System software (SAS Institute, Cary, NC, USA, Version 9.2) and Duncan was used to measuring significant differences among treatment means at $p<0.05$. The Pearson correlation coefficient was used to measure relationships between germination and physiological traits by using SAS software vr.9.2.

\section{Results}

\section{Germination parameters}

The effects of genotypes, salinity, and priming as well as interaction genotype $\times$ salinity $\times$ priming was significant on GP, MGT, GR, GU, RL, PL, SL, and SVI. Salinity, in $12 \mathrm{dS} / \mathrm{m}$ level, reduced GP, GR, RL, PL, SL, and SVI in different sugar beet genotypes but increased MGT and GU (Table 1). Results indicated that a moderate level of salinity $(5 \mathrm{dS} / \mathrm{m})$ had the highest seedling growth parameters such as RL, PL, SL, and SVI compared to the non-stress conditions. In other words, $\mathrm{NaCl}$ concentration up to $5 \mathrm{dS} / \mathrm{m}$ was caused by increases seedling growth parameters (Table 1). In general, the results showed that seed priming increased the mean of germination parameters compared to unprimed seeds (Table 1). For example, the hydro-priming of 'Paya' genotype seeds resulted in the highest GP (95\%) under high level of salinity (12 dS/m) which $11.84 \%$ increase compared to the control treatment (Table 2).

As shown in Table 2, in the interaction effects of genotype, salinity, and priming, the highest MGT was related to seed priming with $\mathrm{ZnSO}_{4}$ in 'Sina' and 'Aria' genotypes under non-stress condition (3.86 and 3.9 days, respectively). On the other hand, the lowest MGT was found in the hydro- and osmopriming of 'Paya' genotype under high level of salinity ( 0.155 and 0.09 days).

Results indicated that the hydropriming of 'Paya' sugar beet seeds increase GR (84.9\%) under high level of salinity compared to the control treatment. Osmopriming by $\mathrm{ZnSO}_{4}$ resulted in the lowest GR ( 0.26 seed per day) in 'Sina' genotype under non-stress conditions (Table 2).

Results indicated that the salinity decreases GU, but priming treatment increases the mean of these traits (Table 1). According to the results of interaction effects (Table 2), GU ranged from 0.14 to 0.715 . The highest GU was related to primed G2 ('Sina' genotype) seed by $\mathrm{ZnSO}_{4}$ under non-stress conditions, and the lowest of these traits was in G3 ('Paya' genotype) under high level of salinity and all priming treatments.

The results of ANOVA showed that the genotype, salinity, and priming significantly affected seedling growth parameters such as RL, PL, SL, and SVI. In terms of seedling growth parameters, 'Aria' genotype had a significant advantage over other genotypes (Table 1). In interaction effects, the highest RL ( $88.75 \mathrm{~mm})$ and SL $(144.0 \mathrm{~mm})$ were related to osmopriming of 'Paya' genotype seeds under $5 \mathrm{dS} / \mathrm{m}$ salinity, while the highest PL $(67.0 \mathrm{~mm})$ and SVI (20.91) was found in hydropriming of G5 genotype ('Aria') seeds under $5 \mathrm{dS} / \mathrm{m}$. The results indicated that the lowest means of these traits was achieved in unprimed 'Paya' genotype seed under high level of salinity (Table 2). 
Table 1. Effect of different levels of salinity stress $(0,2,5$, and $12 \mathrm{dS} / \mathrm{m})$ and priming treatments on germination indices of sugar beet (Beta vulgaris L.) genotypes

\begin{tabular}{|c|c|c|c|c|c|c|c|c|}
\hline \multirow{2}{*}{ Treatments } & GP & MGT & GR & GU & $\mathrm{RL}$ & $\mathrm{PL}$ & SL & SVI \\
\hline & $(\%)$ & (day) & (seed/day) & & \multicolumn{3}{|c|}{$(\mathrm{mm})$} & \\
\hline \multicolumn{9}{|c|}{ Genotypes (G) } \\
\hline G1 & $71.14 \mathrm{~b}$ & $1.26 \mathrm{~d}$ & $1.38 \mathrm{~b}$ & $0.19 \mathrm{~d}$ & $37.56 \mathrm{~b}$ & $38.11 \mathrm{a}$ & $75.67 \mathrm{~b}$ & $10.14 \mathrm{~b}$ \\
\hline G2 & $55.62 \mathrm{e}$ & $2.10 \mathrm{~b}$ & $0.67 \mathrm{c}$ & $0.30 \mathrm{a}$ & $42.22 \mathrm{a}$ & $36.75 \mathrm{a}$ & $78.97 \mathrm{ab}$ & $9.87 \mathrm{bc}$ \\
\hline G3 & $86.66 \mathrm{a}$ & $0.63 \mathrm{e}$ & $2.44 \mathrm{a}$ & $0.15 \mathrm{e}$ & $37.31 \mathrm{~b}$ & $31.40 \mathrm{~b}$ & $68.71 \mathrm{c}$ & $9.31 \mathrm{c}$ \\
\hline G4 & $64.89 \mathrm{c}$ & $1.94 \mathrm{c}$ & $0.63 c$ & $0.23 c$ & $18.29 \mathrm{c}$ & $28.08 \mathrm{c}$ & $46.37 \mathrm{~d}$ & $6.82 \mathrm{~d}$ \\
\hline G5 & $59.27 \mathrm{~d}$ & $2.56 \mathrm{a}$ & $0.44 \mathrm{~d}$ & $0.28 \mathrm{~b}$ & $41.87 \mathrm{a}$ & $38.08 \mathrm{a}$ & $79.95 \mathrm{a}$ & $12.05 \mathrm{a}$ \\
\hline \multicolumn{9}{|c|}{ Salinity levels (S) (dS/m) } \\
\hline Control & $81.50 \mathrm{a}$ & $0.93 \mathrm{~d}$ & $2.37 \mathrm{a}$ & $0.17 \mathrm{~d}$ & $18.26 \mathrm{~d}$ & $20.46 \mathrm{c}$ & $38.73 \mathrm{~d}$ & $5.38 \mathrm{c}$ \\
\hline 2 & $72.41 \mathrm{~b}$ & $1.57 \mathrm{c}$ & $0.82 \mathrm{~b}$ & $0.20 \mathrm{c}$ & $36.23 c$ & $37.57 \mathrm{~b}$ & $73.80 \mathrm{c}$ & $10.59 \mathrm{~b}$ \\
\hline 5 & $61.75 \mathrm{c}$ & $1.96 \mathrm{~b}$ & $0.72 \mathrm{~b}$ & $0.25 \mathrm{~b}$ & $44.73 \mathrm{a}$ & $43.07 \mathrm{a}$ & $87.80 \mathrm{a}$ & $12.05 \mathrm{a}$ \\
\hline 12 & $54.41 \mathrm{~d}$ & $2.33 \mathrm{a}$ & $0.54 \mathrm{c}$ & $0.30 \mathrm{a}$ & $42.58 \mathrm{~b}$ & $36.83 \mathrm{~b}$ & $79.41 \mathrm{~b}$ & $10.53 \mathrm{~b}$ \\
\hline \multicolumn{9}{|c|}{ Priming $(\mathrm{P})$} \\
\hline Unprimed seed (control) & $64.06 \mathrm{~b}$ & $1.23 \mathrm{c}$ & $0.96 \mathrm{~b}$ & $0.18 \mathrm{c}$ & $10.27 \mathrm{c}$ & $19.17 \mathrm{c}$ & $29.45 c$ & $4.27 c$ \\
\hline Hydro-priming & $80.37 \mathrm{a}$ & $1.75 \mathrm{~b}$ & $1.37 \mathrm{a}$ & $0.24 \mathrm{~b}$ & $51.42 \mathrm{a}$ & $44.06 \mathrm{a}$ & $95.48 \mathrm{a}$ & $13.16 \mathrm{a}$ \\
\hline $\mathrm{ZnSO}_{4}$ & $58.12 \mathrm{c}$ & $2.12 \mathrm{a}$ & $1.01 \mathrm{~b}$ & $0.28 \mathrm{a}$ & $44.66 \mathrm{~b}$ & $40.22 \mathrm{~b}$ & $84.88 \mathrm{~b}$ & $11.48 \mathrm{a}$ \\
\hline \multicolumn{9}{|c|}{ Statistics } \\
\hline$G \times S$ & ** & *** & ** & ** & ** & ** & ** & ** \\
\hline $\mathrm{G} \times \mathrm{P}$ & ** & *** & ** & $* *$ & $* *$ & *** & ** & *** \\
\hline $\mathrm{S} \times \mathrm{P}$ & $* *$ & ** & ** & ** & ** & ** & ** & ** \\
\hline $\mathrm{G} \times \mathrm{S} \times \mathrm{P}$ & ** & ** & ** & ** & ** & ** & ** & ** \\
\hline
\end{tabular}

Means followed by the same letter in each column are not significantly different according to Duncan test at $5 \%$ level

**: significant at $\alpha=\alpha=0.01$.

G1: Shokofa; G2: Sina; G3: Paya; G4: Turbata; G5: Aria

GP: germination percentage; MGT: Mean time germination; GR: germination rate; GU: germination uniformity; RL:

radical length; PL: plumule length; SL: seedling length; SVI: seedling vigor index

Table 2. Interaction effects of salinity and priming on germination indices of sugar beet (Beta vulgaris L.) genotypes

\begin{tabular}{|c|c|c|c|c|c|c|c|c|c|c|}
\hline Genotypes & Salinity & Priming & GP (\%) & MGT (day) & GR (seed/day) & GU & $\mathrm{RL}(\mathrm{mm})$ & PL (mm) & $\mathrm{SL}(\mathrm{mm})$ & SVI \\
\hline \multirow{12}{*}{ G1 } & \multirow{3}{*}{ S1 } & C & 75 i.l & $1.44 \mathrm{n} . \mathrm{r}$ & $0.7 \mathrm{i} . \mathrm{m}$ & 0.19 l.r & 13.5 o.t & $30 \mathrm{~m} \cdot \mathrm{t}$ & 43.5 q.u & $6.21 \mathrm{p} . \mathrm{s}$ \\
\hline & & $\mathrm{H}$ & $60 \mathrm{opq}$ & $1.42 \mathrm{n} . \mathrm{r}$ & $0.7 \mathrm{i} . \mathrm{m}$ & 0.215 j.p & $62.5 \mathrm{de}$ & 46.25 g.k & 108.75 d.h & 13.85 f.k \\
\hline & & $S$ & $43.75 \mathrm{uv}$ & $2.79 \mathrm{efg}$ & $0.36 \mathrm{~lm}$ & 0.315 e.h & $52.5 \mathrm{fgh}$ & $36.25 \mathrm{k.q}$ & 88.75 j.m & 12.13 j.m \\
\hline & \multirow{3}{*}{ S2 } & $\mathrm{C}$ & $71.25 \mathrm{klm}$ & $1.22 \mathrm{q.v}$ & 0.82 g.m & 0.19 l.r & 6 tuv & $29 \mathrm{n} . \mathrm{t}$ & 35 s.x & 4.72 r.u \\
\hline & & $\mathrm{H}$ & 55 qrs & $1.84 \mathrm{k} . \mathrm{n}$ & $0.54 \mathrm{~lm}$ & $0.235 \mathrm{jkl}$ & $65 \mathrm{de}$ & 58.25 a.e & $123.25 \mathrm{bcd}$ & 15.97 c.f \\
\hline & & $S$ & 60 opq & $2.02 \mathrm{j} . \mathrm{m}$ & $0.5 \mathrm{~lm}$ & 0.23 j.m & $62.5 \mathrm{de}$ & $62.75 a b c$ & $125.25 \mathrm{bc}$ & $17.57 \mathrm{bcd}$ \\
\hline & \multirow{3}{*}{ S5 } & $\mathrm{C}$ & $83.75 \mathrm{~d} . \mathrm{g}$ & 1.01 r.y & 1.05 f.m & 0.17 n.r & 10.5 q.v & $33.5 \mathrm{~m} . \mathrm{r}$ & 44 q.t & $6.25 \mathrm{p} . \mathrm{s}$ \\
\hline & & $\mathrm{H}$ & $72.5 \mathrm{j} . \mathrm{m}$ & 1.24 q.u & 0.82 g.m & 0.185 l.r & $62.5 \mathrm{de}$ & 47.6 e.k & 110.1 c.g & $14.83 \mathrm{e.i}$ \\
\hline & & $S$ & 75 i.l & 1.05 r.y & $0.95 \mathrm{f} . \mathrm{m}$ & $0.18 \mathrm{l} . \mathrm{r}$ & $57 \mathrm{efg}$ & 57.25 a.g & $114.25 \mathrm{c.f}$ & $15.33 \mathrm{~d} . \mathrm{g}$ \\
\hline & \multirow{3}{*}{ S12 } & $\mathrm{C}$ & 85 c.f & 0.75 v.y & $1.43 \mathrm{e} . \mathrm{i}$ & $0.16 \mathrm{pqr}$ & 4.5 tuv & $6.5 \mathrm{yz}$ & $11 \mathrm{z}$ & $1.51 \mathrm{wx}$ \\
\hline & & $\mathrm{H}$ & 83.75 d.g & $0.215 \mathrm{z}$ & $5.66 c$ & $0.15 \mathrm{r}$ & $35 \mathrm{j}$ & $34 \mathrm{~m} \cdot \mathrm{r}$ & 69 no & 8.74 nop \\
\hline & & $S$ & 88.75 a.e & $0.165 \mathrm{z}$ & $3.08 \mathrm{~d}$ & $0.145 \mathrm{r}$ & $19.25 \mathrm{~m} . \mathrm{q}$ & 16 u.y & 35.25 s.x & 4.63 r.u \\
\hline \multirow{12}{*}{ G2 } & \multirow{3}{*}{ S1 } & $\mathrm{C}$ & $58.75 \mathrm{pqr}$ & 1.79 l.o & $0.56 \mathrm{klm}$ & 0.225 j.n & 8.5 r.v & $30.5 \mathrm{~m} . \mathrm{t}$ & 39 q.v & 5.17 q.t \\
\hline & & $\mathrm{H}$ & $22.5 \mathrm{x}$ & $3.28 \mathrm{bcd}$ & $0.3 \mathrm{~lm}$ & $0.475 c$ & $65.75 \mathrm{de}$ & 37 k.o & $102.75 \mathrm{f.j}$ & $10.83 \mathrm{lmn}$ \\
\hline & & $S$ & $11.25 \mathrm{y}$ & $3.86 \mathrm{a}$ & $0.26 \mathrm{~m}$ & $0.715 \mathrm{a}$ & $63.5 \mathrm{de}$ & $32.5 \mathrm{~m} . \mathrm{s}$ & 96 g.k & $7.62 \mathrm{opq}$ \\
\hline & \multirow{3}{*}{ S2 } & C & 77.5 g.k & 1.16 q.v & 0.86 g.m & $0.18 \mathrm{l} . \mathrm{r}$ & $8.5 \mathrm{r.v}$ & 23.5 r.w & 32 s.y & $4.48 \mathrm{s.v}$ \\
\hline & & $\mathrm{H}$ & $43.75 \mathrm{uv}$ & $2.69 \mathrm{fgh}$ & $0.375 \mathrm{~lm}$ & 0.315 e.h & $81.25 \mathrm{ab}$ & $51.25 \mathrm{~d} . j$ & $132.5 \mathrm{ab}$ & $18.01 \mathrm{bc}$ \\
\hline & & $S$ & $20 x$ & $3.55 \mathrm{ab}$ & $0.28 \mathrm{~lm}$ & $0.535 \mathrm{~b}$ & $60 \mathrm{def}$ & $41.25 \mathrm{j} . \mathrm{m}$ & $101.25 \mathrm{f} . \mathrm{j}$ & $10.86 \mathrm{lmn}$ \\
\hline & \multirow{3}{*}{ S5 } & $\mathrm{C}$ & 88.75 a.e & 0.79 u.y & $1.51 \mathrm{e} . \mathrm{h}$ & $0.16 \mathrm{pqr}$ & 9.5 r.v & 26 p.v & 35.5 s.x & 5 r.u \\
\hline & & $\mathrm{H}$ & $52.5 \mathrm{rst}$ & 2.25 i.k & $0.44 \mathrm{~lm}$ & $0.26 \mathrm{ijk}$ & $62 \mathrm{de}$ & 59.25 a.d & 121.25 b.e & $16.63 \mathrm{cde}$ \\
\hline & & $S$ & $50 \mathrm{stu}$ & 2.26 h.k & $0.44 \mathrm{~lm}$ & 0.265 h.k & $61.25 \mathrm{de}$ & 45.25 h.k & 106.5 e.i & 14.33 e.j \\
\hline & \multirow{3}{*}{ S12 } & $\mathrm{C}$ & $91.25 \mathrm{abc}$ & 0.925 t.y & $1.08 \mathrm{f} .1$ & $0.16 \mathrm{pqr}$ & 6.5 tuv & 13.5 w.z & 20 w.z & 2.93 t.x \\
\hline & & $\mathrm{H}$ & 83.75 d.g & 0.675 wxy & 150 e.h & $0.16 \mathrm{pqr}$ & 50 ghi & 43 i.l & 93 h.l & 12.48 i.m \\
\hline & & $S$ & $67.5 \mathrm{mn}$ & $2 \mathrm{j} . \mathrm{m}$ & $0.5 \mathrm{~lm}$ & 0.215 j.p & $30 \mathrm{jkl}$ & $38 \mathrm{k} . \mathrm{n}$ & 68 nop & $10.07 \mathrm{mno}$ \\
\hline \multirow{4}{*}{ G3 } & \multirow{3}{*}{ S1 } & $\mathrm{C}$ & $83.75 \mathrm{~d} . \mathrm{g}$ & 1.01 r.y & 1 f.m & 0.165 o.r & $12 \mathrm{p.v}$ & 19.5 t.x & 31.5 s.y & $4.41 \mathrm{s.v}$ \\
\hline & & $\mathrm{H}$ & 77.5 g.k & 1.31 p.t & 0.87 g.m & 0.18 l.r & $82.5 \mathrm{ab}$ & $54 \mathrm{c.i}$ & $136.5 \mathrm{ab}$ & $19.31 \mathrm{ab}$ \\
\hline & & $S$ & 75 i.l & 1.12 r.w & 1.04 f.m & 0.185 l.r & 50 ghi & 45.5 h.k & 95.5 g.k & $12.83 \mathrm{~g} .1$ \\
\hline & S2 & $\mathrm{C}$ & 87.5 b.e & $0.605 y$ & $1.69 \mathrm{ef}$ & $0.155 \mathrm{qr}$ & 11 q.v & 21.5 s.x & 32.5 s.y & 4.44 s.v \\
\hline
\end{tabular}




\begin{tabular}{|c|c|c|c|c|c|c|c|c|c|c|}
\hline & & $\mathrm{H}$ & $92.5 \mathrm{ab}$ & $0.19 \mathrm{z}$ & $2.07 \mathrm{e}$ & $0.145 \mathrm{r}$ & $80 \mathrm{~b}$ & $53.6 \mathrm{c.i}$ & $133.6 \mathrm{ab}$ & $17.49 \mathrm{bcd}$ \\
\hline & & $S$ & $80 \mathrm{f} . \mathrm{i}$ & 1 r.y & $1.05 \mathrm{f} . \mathrm{m}$ & 0.17 n.r & $63.75 \mathrm{de}$ & 57.5 a.f & 121.25 b.e & $16.55 \mathrm{cde}$ \\
\hline & & $\mathrm{C}$ & 90 a.d & 0.755 v.y & 1.34 e.k & $0.155 \mathrm{qr}$ & $10.5 \mathrm{q} . \mathrm{v}$ & 17.5 u.y & $28 \mathrm{t} . \mathrm{z}$ & 3.96 s.w \\
\hline & S5 & $\mathrm{H}$ & $92.5 \mathrm{ab}$ & $0.09 \mathrm{z}$ & 1.48 e.i & $0.14 \mathrm{r}$ & 5 tuv & 16.5 u.y & 21.5 w.z & 2.75 t.x \\
\hline & & $S$ & 83.75 d.g & 0.755 v.y & 1.37 e.j & 0.165 o.r & $88.75 \mathrm{a}$ & 55.25 b.h & $144 \mathrm{a}$ & $19.5 \mathrm{ab}$ \\
\hline & & $\mathrm{C}$ & $91.25 \mathrm{abc}$ & $0.64 x y$ & $1.57 \mathrm{efg}$ & $0.15 \mathrm{r}$ & $3 v$ & $4 z$ & $7 z$ & $0.98 \mathrm{x}$ \\
\hline & $\mathrm{S} 12$ & $\mathrm{H}$ & $95 \mathrm{a}$ & $0.155 \mathrm{z}$ & $7.3 \mathrm{a}$ & $0.14 \mathrm{r}$ & $35 \mathrm{j}$ & 19.5 t.x & $54.5 \mathrm{opq}$ & $7.15 \mathrm{pqr}$ \\
\hline & & $S$ & $91.25 \mathrm{abc}$ & $0.09 \mathrm{z}$ & $6.85 \mathrm{~b}$ & $0.145 \mathrm{r}$ & 6.25 tuv & $12.5 \mathrm{w} . \mathrm{z}$ & $18.75 \mathrm{xyz}$ & 2.38 u.x \\
\hline \multirow{12}{*}{ G4 } & \multirow{3}{*}{ S1 } & $\mathrm{C}$ & $72.5 \mathrm{j} . \mathrm{m}$ & 1.46 n.r & $0.68 \mathrm{i} . \mathrm{m}$ & 0.195 l.r & 12 p.v & $15 \mathrm{w} . \mathrm{z}$ & 27 u.z & 3.78 s.w \\
\hline & & $\mathrm{H}$ & $45 \mathrm{uv}$ & $3.36 \mathrm{bc}$ & $0.29 \mathrm{~lm}$ & $0.355 \mathrm{de}$ & $24 \operatorname{lmn}$ & $30.5 \mathrm{~m} . \mathrm{t}$ & $54.5 \mathrm{opq}$ & 8.58 nop \\
\hline & & $S$ & 46.25 tuv & $3.42 \mathrm{bc}$ & $0.29 \mathrm{~lm}$ & $0.355 \mathrm{de}$ & $26 \mathrm{klm}$ & $47 \mathrm{f} . \mathrm{k}$ & $73 \mathrm{mn}$ & 11.86 j.m \\
\hline & \multirow{3}{*}{ S2 } & $\mathrm{C}$ & 76.25 h.l & $1.41 \mathrm{n} . \mathrm{r}$ & 0.72 h.m & 0.185 l.r & $7.5 \mathrm{s.v}$ & $13 \mathrm{w} . \mathrm{z}$ & 20.5 w.z & 2.92 t.x \\
\hline & & $\mathrm{H}$ & $58.75 \mathrm{pqr}$ & 2.07 i.l & $0.48 \mathrm{~lm}$ & $0.235 \mathrm{jkl}$ & $44 \mathrm{i}$ & 37.5 k.o & 81.5 k.n & $11.34 \mathrm{klm}$ \\
\hline & & $S$ & $45 \mathrm{uv}$ & $2.64 \mathrm{fgh}$ & $0.39 \mathrm{~lm}$ & $0.3 \mathrm{f} . \mathrm{i}$ & $33.5 \mathrm{jk}$ & 58.5 a.e & 92 i.l & 12.55 h.m \\
\hline & \multirow{3}{*}{ S5 } & $\mathrm{C}$ & 82.5 e.h & 1.35 o.t & 0.74 h.m & 0.18 l.r & $7.5 \mathrm{s.v}$ & $10.5 \mathrm{yxz}$ & $18 \mathrm{yz}$ & 2.67 t.x \\
\hline & & $\mathrm{H}$ & 60 opq & 1.63 l.q & 0.61 j.m & 0.22 j.o & 21.51 .0 & 26.5 o.u & 48 qrs & 6.32 p.s \\
\hline & & $S$ & 63.75 nop & $2.87 \mathrm{~d} . \mathrm{g}$ & $0.35 \mathrm{~lm}$ & 0.27 hij & 20.5 m.p & $52.5 \mathrm{c.i}$ & $73 \mathrm{mn}$ & 12.52 h.m \\
\hline & \multirow{3}{*}{ S12 } & $\mathrm{C}$ & $78.75 \mathrm{f.j}$ & 1.03 r.y & 1.03 f.m & 0.18 l.r & $3.5 \mathrm{uv}$ & $4 z$ & $7.5 \mathrm{z}$ & $1.04 \mathrm{x}$ \\
\hline & & $\mathrm{H}$ & 75 i.l & 1.09 r.x & 0.92 f.m & 0.18 l.r & 12.5 p.t & $34 \mathrm{~m} \cdot \mathrm{r}$ & $46.5 \mathrm{qrs}$ & 6.28 p.s \\
\hline & & $S$ & 75 i.l & 0.955 s.y & 1.07 f.m & $0.175 \mathrm{~m} . \mathrm{r}$ & $7 \mathrm{s.v}$ & $8 \mathrm{yz}$ & $15 \mathrm{yz}$ & $1.97 \mathrm{vwx}$ \\
\hline \multirow{12}{*}{ G5 } & \multirow{3}{*}{ S1 } & $\mathrm{C}$ & $71.25 \mathrm{klm}$ & 1.93 j.m & $0.52 \mathrm{~lm}$ & $0.21 \mathrm{k} . \mathrm{q}$ & $22.5 \mathrm{lmn}$ & $30.5 \mathrm{~m} . \mathrm{t}$ & $53 \mathrm{pqr}$ & 7.93 op \\
\hline & & $\mathrm{H}$ & $42.5 \mathrm{v}$ & $2.85 \mathrm{~d} . \mathrm{g}$ & $0.335 \mathrm{~lm}$ & $0.325 \mathrm{efg}$ & $75.5 \mathrm{bc}$ & 59 a.d & $134.5 \mathrm{ab}$ & $18.35 b c$ \\
\hline & & $S$ & $31.25 \mathrm{w}$ & $3.9 \mathrm{a}$ & $0.265 \mathrm{~lm}$ & $0.515 b c$ & $68 \mathrm{~cd}$ & 39 k.n & 107 d.i & 15.1 d.h \\
\hline & \multirow{3}{*}{ S2 } & $\mathrm{C}$ & $78.75 \mathrm{f.j}$ & $2.47 \mathrm{ghi}$ & $0.46 \mathrm{~lm}$ & 0.23 j.m & 21.5 l.o & 25 q.v & $46.5 \mathrm{qrs}$ & 8.52 nop \\
\hline & & $\mathrm{H}$ & 46.25 tuv & $3.42 \mathrm{bc}$ & $0.29 \mathrm{~lm}$ & $0.35 \mathrm{def}$ & $67 \mathrm{~d}$ & $66 \mathrm{ab}$ & $133 \mathrm{ab}$ & $21.5 \mathrm{a}$ \\
\hline & & $S$ & $33.75 \mathrm{w}$ & 3.21 b.e & $0.31 \mathrm{~lm}$ & $0.39 \mathrm{~d}$ & $59.5 \mathrm{def}$ & 47.5 e.k & 107 d.i & $13.92 \mathrm{f} . \mathrm{k}$ \\
\hline & \multirow{3}{*}{ S5 } & $\mathrm{C}$ & $83.75 \mathrm{~d} . \mathrm{g}$ & $1.6 \mathrm{~m} . \mathrm{q}$ & $0.62 \mathrm{j} . \mathrm{m}$ & 0.185 l.r & $160 . s$ & 17 u.y & 33 s.y & 5.13 q.t \\
\hline & & $\mathrm{H}$ & $52.5 \mathrm{rst}$ & 3.06 c.f & $0.315 \mathrm{~lm}$ & 0.305 e.i & $64 \mathrm{de}$ & $67 a$ & $131 \mathrm{ab}$ & $20.91 \mathrm{a}$ \\
\hline & & $S$ & $55 \mathrm{qrs}$ & 3 c.f & $0.325 \mathrm{~lm}$ & 0.295 ghi & $47 \mathrm{hi}$ & 32 l.s & $79 \operatorname{lmn}$ & 12.72 h.l \\
\hline & \multirow{3}{*}{ S12 } & $\mathrm{C}$ & $80 \mathrm{f} . \mathrm{i}$ & $1.21 \mathrm{q.v}$ & 0.83 g.m & 0.18 l.r & $11 \mathrm{q} \cdot \mathrm{v}$ & $13.5 \mathrm{w} . \mathrm{z}$ & 24.5 v.z & 3.49 t.x \\
\hline & & $\mathrm{H}$ & $70 \mathrm{~lm}$ & $2.33 \mathrm{hij}$ & $0.44 \mathrm{~lm}$ & $0.23 \mathrm{j} . \mathrm{m}$ & $33.5 \mathrm{jk}$ & $40.5 \mathrm{j} . \mathrm{n}$ & $74 \mathrm{mn}$ & 11.93 j.m \\
\hline & & $S$ & $66.25 \mathrm{mno}$ & $1.751 . \mathrm{p}$ & 0.57 j.m & $0.21 \mathrm{k.q}$ & 17 o.r & 20 t.x & 37 r.w & 5.17 q.t \\
\hline
\end{tabular}

Means followed by the same letter in each column are not significantly different according to Duncan test at $5 \%$ level G1: Shokofa; G2: Sina; G3: Paya; G4: Turbata; G5: Aria

C: control (non-priming); H: Hyrdopriming; S: ZnSO 4

GP: germination percentage; MGT: Mean time germination; GR: germination rate; GU: germination uniformity; RL:

radical length; PL: plumule length; SL: seedling length; SVI: seedling vigor index

\section{Physiological and biochemical characteristics}

Genotypes, salinity, and priming as well as interaction of them significantly affected RWC, chlorophyll a, b, and total, carotenoids, anthocyanin, SOD, protein, proline, and sugar contents (Table 3). Salinity stress decreased RWC, while priming increased it. The highest RWC was related to 'Turbata' seeds primed by $\mathrm{ZnSO}_{4}$ under 2 and $5 \mathrm{dS} / \mathrm{m}$ salinity levels (90.45 and 85.7\%) which increased by $36.5 \%$ and 33.0\%, respectively, compared to the control treatment. Also, in 'Aria' genotype, all priming treatment showed the highest RWC under non-stress conditions (Table 4).

High level of salinity stress was caused by decreasing photosynthesis pigments such as chlorophyll-a, b, and total as well as carotenoid contents by 43.9, 31.9, 39.9, and 20.4\% compared to the control treatment $(0$ $\mathrm{d} S / \mathrm{m})$. Hydropriming of 'Sina' seeds showed the highest chlorophyll-a, total, and carotenoids under non-stress conditions $(22.89,31.65$, and $2116.6 \mu \mathrm{g} / \mathrm{g} \mathrm{FW})$. Also, hydropriming by increases chlorophyll b content led to the modulation of the negative effects of high salinity stress $(12 \mathrm{dS} / \mathrm{m})$ (Table 4$)$.

As shown in Table 4, $\mathrm{ZnSO}_{4}$ priming of 'Shokofa' seeds significantly increased (79.3\%) the anthocyanin content under salinity stressed plant compared to the non-stress conditions. The lowest mean of anthocyanin content was achieved in the hydropriming of 'Shokofa' (1.84 $\mu \mathrm{g} / \mathrm{g} \mathrm{FW})$ and unprimed of 'Sina' (1.91 $\mu \mathrm{g} / \mathrm{g} \mathrm{FW})$ seeds under non-stress conditions.

Results indicated that salinity stress significantly increases SOD activity (Table 3). Based on these findings, osmopriming of 'Shokofa' genotype had the highest SOD activity $(90.3 \mathrm{U} / \mathrm{mg}$ protein) under high 
level of salinity $(12 \mathrm{dS} / \mathrm{m})$ and the lowest mean $(9.9 \mathrm{U} / \mathrm{mg}$ protein $)$ was related to 'Turbata' genotype under unprimed and non-stress conditions (Table 4).

Genotype, salinity, and priming treatments significantly affected seedling protein content. Salinity decreased protein content while seed priming increased compared to the control treatment (Table 3). The highest protein content (12.35\%) was related to 'Aria' genotype under unprimed and non-stress conditions, whereas the lowest this trait (2.1\%) was in unprimed seeds of 'Sina' genotype under high level of salinity (12 $\mathrm{dS} / \mathrm{m})$ (Table 4).

Results showed that the main and interaction effects of genotype, salinity, and priming were significant on proline content (Table 3). Results illustrated that the salinity and priming treatments significantly increases seedling proline content, so that the highest proline content $(2.15 \mu \mathrm{mol} / \mathrm{g} \mathrm{FW})$ was achieved in the osmopriming by $\mathrm{ZnSO}_{4}$ of 'Shokofa' genotype under severe salinity treatment, and the lowest mean was related to the unprimed of 'Sina' seeds under $2 \mathrm{dS} / \mathrm{m}$ salinity $(0.337 \mu \mathrm{mol} / \mathrm{g} \mathrm{FW})$ (Table 4$)$.

Significant sugar accumulations were observed in sugar beet seedlings at all salinity concentrations and priming treatments (Table 3). In the interaction effects, the highest sugar content was found 'Shokofa' seed primed by $\mathrm{ZnSO}_{4}$ under high level of salinity $(33.2 \mathrm{mg} / \mathrm{g} \mathrm{FW})$. The sugar accumulation at $12 \mathrm{dS} / \mathrm{m}$ in 'Shokofa' rose to about 6-fold that of the control treatment (Table 4). On the other hand, the lowest mean of this trait was observed in the unprimed of 'Turbata' genotype under non-stress conditions ( $4.54 \mathrm{mg} / \mathrm{g} \mathrm{FW}$ ) (Table 4).

Table 3. Effect of different levels of salinity stress $(0,2,5$, and $12 \mathrm{dS} / \mathrm{m})$ and priming treatments on physiological characteristics of sugar beet (Beta vulgaris L.) genotypes

\begin{tabular}{|c|c|c|c|c|c|c|c|c|c|c|}
\hline \multirow{2}{*}{ Treatments } & RWC & Chl a & $\mathrm{Chlb}$ & $\begin{array}{c}\text { Total } \\
\text { Chl }\end{array}$ & Carotenoids & Anthocyanin & $\begin{array}{l}\text { SOD } \\
\text { activity }\end{array}$ & $\begin{array}{l}\text { Protein } \\
\text { content }\end{array}$ & $\begin{array}{l}\text { Proline } \\
\text { content }\end{array}$ & $\begin{array}{l}\text { Sugar } \\
\text { content }\end{array}$ \\
\hline & $(\%)$ & \multicolumn{5}{|c|}{$(\mu \mathrm{g} / \mathrm{g} F W)$} & $\begin{array}{c}(\mathrm{U} / \mathrm{mg} \\
\text { protein })\end{array}$ & $(\%)$ & $\begin{array}{c}(\mu \mathrm{mol} / \mathrm{g} \\
\mathrm{FW})\end{array}$ & $\begin{array}{c}(\mathrm{mg} / \mathrm{g} \\
\mathrm{FW})\end{array}$ \\
\hline \multicolumn{11}{|c|}{ Genotypes (G) } \\
\hline G1 & $52.15 b$ & $6.42 \mathrm{~d}$ & $2.59 \mathrm{~d}$ & $9.02 \mathrm{~d}$ & $5.65 \mathrm{~d}$ & $5.16 \mathrm{a}$ & $25.35 \mathrm{a}$ & $6.80 \mathrm{~d}$ & $0.68 \mathrm{a}$ & $10.13 \mathrm{a}$ \\
\hline G2 & $46.23 \mathrm{~b}$ & $9.09 \mathrm{c}$ & $3.65 c$ & $12.75 \mathrm{c}$ & $7.82 \mathrm{c}$ & $2.63 \mathrm{e}$ & $19.76 \mathrm{c}$ & $7.24 \mathrm{c}$ & $0.55 \mathrm{c}$ & $8.15 \mathrm{c}$ \\
\hline G3 & $42.43 \mathrm{~b}$ & $5.01 \mathrm{e}$ & $1.95 \mathrm{e}$ & $6.96 \mathrm{e}$ & $5.07 \mathrm{e}$ & $3.02 \mathrm{~d}$ & $21.35 \mathrm{~b}$ & $6.79 \mathrm{~d}$ & $0.59 \mathrm{~b}$ & $8.72 \mathrm{~b}$ \\
\hline G4 & $66.73 \mathrm{a}$ & $9.53 \mathrm{a}$ & $4.49 \mathrm{a}$ & $14.02 \mathrm{a}$ & $9.36 \mathrm{~b}$ & $4.83 \mathrm{~b}$ & $17.96 \mathrm{~d}$ & $7.99 \mathrm{~b}$ & $0.51 \mathrm{~d}$ & $7.28 \mathrm{e}$ \\
\hline G5 & $69.65 \mathrm{a}$ & $9.26 \mathrm{~b}$ & $4.02 \mathrm{~b}$ & $13.28 \mathrm{~b}$ & $9.51 \mathrm{a}$ & $4.63 \mathrm{c}$ & $17.84 \mathrm{~d}$ & $8.56 \mathrm{a}$ & $0.50 \mathrm{~d}$ & $7.52 \mathrm{~d}$ \\
\hline \multicolumn{11}{|c|}{ Salinity levels (S) (dS/m) } \\
\hline Control & $86.33 \mathrm{a}$ & $9.92 \mathrm{a}$ & $4.20 \mathrm{a}$ & $14.12 \mathrm{a}$ & $8.32 \mathrm{a}$ & $3.39 \mathrm{~d}$ & $13.04 \mathrm{~d}$ & $8.19 \mathrm{a}$ & $0.40 \mathrm{~d}$ & $5.68 \mathrm{~d}$ \\
\hline 2 & $62.77 \mathrm{~b}$ & $8.67 \mathrm{~b}$ & $3.38 \mathrm{~b}$ & $12.05 \mathrm{~b}$ & $7.81 \mathrm{~b}$ & $4.84 \mathrm{a}$ & $15.39 c$ & $8.00 \mathrm{~b}$ & $0.46 \mathrm{c}$ & $6.63 c$ \\
\hline 5 & $55.19 \mathrm{~b}$ & $7.31 \mathrm{c}$ & $2.91 \mathrm{c}$ & $10.17 \mathrm{c}$ & $7.19 \mathrm{c}$ & $3.81 \mathrm{c}$ & $19.68 \mathrm{~b}$ & $7.02 \mathrm{c}$ & $0.55 \mathrm{~b}$ & $8.13 \mathrm{~b}$ \\
\hline 12 & $43.06 \mathrm{~b}$ & $5.56 \mathrm{~d}$ & $2.86 \mathrm{c}$ & $8.48 \mathrm{~d}$ & $6.62 \mathrm{~d}$ & $4.18 \mathrm{~b}$ & $33.70 \mathrm{a}$ & $5.97 \mathrm{~d}$ & $0.87 \mathrm{a}$ & $13.00 \mathrm{a}$ \\
\hline \multicolumn{11}{|c|}{ Priming $(\mathrm{P})$} \\
\hline Control & $57.81 \mathrm{~b}$ & $5.68 \mathrm{c}$ & $2.17 \mathrm{c}$ & $7.85 \mathrm{c}$ & $5.39 c$ & $3.57 \mathrm{c}$ & $16.28 \mathrm{c}$ & $6.76 \mathrm{~b}$ & $0.46 \mathrm{c}$ & $6.76 \mathrm{c}$ \\
\hline $\begin{array}{l}\text { Hydro- } \\
\text { priming }\end{array}$ & $66.51 \mathrm{a}$ & $8.45 \mathrm{~b}$ & $4.30 \mathrm{a}$ & $12.75 \mathrm{~b}$ & $8.68 \mathrm{a}$ & $3.66 \mathrm{~b}$ & $19.81 \mathrm{~b}$ & $7.82 \mathrm{a}$ & $0.55 \mathrm{~b}$ & $8.19 \mathrm{~b}$ \\
\hline $\mathrm{ZnSO}_{4}$ & $61.18 \mathrm{a}$ & $9.46 \mathrm{a}$ & $3.55 \mathrm{~b}$ & $13.01 \mathrm{a}$ & $8.38 \mathrm{~b}$ & $4.94 \mathrm{a}$ & $25.26 \mathrm{a}$ & $7.86 \mathrm{a}$ & $0.68 \mathrm{a}$ & $10.13 \mathrm{a}$ \\
\hline \multicolumn{11}{|c|}{ Statistics } \\
\hline $\mathrm{G} \times \mathrm{S}$ & ** & ** & ** & ** & ** & ** & ** & ** & ** & ** \\
\hline $\mathrm{G} \times \mathrm{P}$ & ** & *** & *** & ** & ** & ** & ** & *** & ** & ** \\
\hline $\mathrm{S} \times \mathrm{P}$ & NS & *** & *** & *** & *** & *** & *** & *** & *** & *** \\
\hline$G \times S \times P$ & ** & *** & ** & ** & ** & ** & ** & *** & ** & ** \\
\hline
\end{tabular}

Means followed by the same letter in each column are not significantly different according to Duncan test at $5 \%$ level NS: non-significant; ${ }^{* *}$ : significant at $\alpha=\alpha=0.01$.

G1: Shokofa; G2: Sina; G3: Paya; G4: Turbata; G5: Aria

Chl a: chlorophyll a; Chl b: chlorophyll b; Total Chl: total chlorophyll; SOD: superoxide dismutase. 
Shokouhian A and Omidi H (2021). Not Bot Horti Agrobo 49(3):12063

Table 4. Interaction effects of salinity and priming on physiological characteristics of sugar beet (Beta vulgaris L.) genotypes

\begin{tabular}{|c|c|c|c|c|c|c|c|c|c|c|c|c|}
\hline Genotypes & Salinity & Priming & $\begin{array}{l}\text { RWC } \\
(\%)\end{array}$ & $\begin{array}{c}\mathrm{Chla} \\
(\mu \mathrm{g} / \mathrm{g} \mathrm{FW})\end{array}$ & $\begin{array}{c}\mathrm{Chl} \mathrm{b} \\
(\mu \mathrm{g} / \mathrm{g} \mathrm{FW})\end{array}$ & $\begin{array}{l}\text { Total Chl } \\
(\mu \mathrm{g} / \mathrm{g} \mathrm{FW})\end{array}$ & $\begin{array}{l}\text { Carotenoid } \\
(\mu \mathrm{g} / \mathrm{g} \mathrm{FW})\end{array}$ & $\begin{array}{c}\text { Anthocyanin } \\
(\mu \mathrm{g} / \mathrm{g} \mathrm{FW})\end{array}$ & $\begin{array}{c}\text { SOD } \\
\text { (U/mg protein) }\end{array}$ & $\begin{array}{c}\text { Protein } \\
(\%)\end{array}$ & $\begin{array}{c}\text { Proline } \\
(\mu \mathrm{mol} / \mathrm{g} \mathrm{FW})\end{array}$ & $\begin{array}{c}\begin{array}{c}\text { Sugar } \\
\text { (mg/g }\end{array} \\
\text { FW) }\end{array}$ \\
\hline \multirow{12}{*}{ G1 } & \multirow{3}{*}{ S1 } & C & $48.5 \mathrm{~h} . \mathrm{n}$ & $7.18 \mathrm{y}$ & $4.37 \mathrm{hi}$ & 11.54 op & $673.5 \mathrm{a}^{*}$ & $2.64 \mathrm{w} \cdot \mathrm{z}$ & $13.1 \mathrm{stu}$ & $8.04 \mathrm{n} \cdot \mathrm{q}$ & 0.399 yza $^{*}$ & $\begin{array}{l}5.62 \\
\text { yza }^{*}\end{array}$ \\
\hline & & $\mathrm{H}$ & 50.65 g.l & $8.24 \mathrm{r}$ & 2.71 p.t & $10.95 \mathrm{qr}$ & $778.3 \mathrm{u}$ & $1.84 \mathrm{z}$ & 11.6 u.x & $8.61 \mathrm{hij}$ & $0.379 \mathrm{z.c}^{*}$ & $\begin{array}{l}5.31 \\
\mathrm{z.c} \mathrm{c}^{*}\end{array}$ \\
\hline & & $S$ & $73.6 \mathrm{bcd}$ & $7.76 \mathrm{w}$ & $3.58 \mathrm{j} . \mathrm{m}$ & $11.33 \mathrm{pq}$ & $730.4 x$ & $2.34 \mathrm{xyz}$ & 16.4 nop & $8.8 \mathrm{gh}$ & $0.487 \mathrm{qrs}$ & $\begin{array}{c}7.12 \\
\text { st }\end{array}$ \\
\hline & \multirow{3}{*}{ S2 } & $\mathrm{C}$ & $55 \mathrm{f.j}$ & $6.94 \mathrm{z}$ & $2.84 \mathrm{n} . \mathrm{s}$ & 9.78 tuv & $583.4 \mathrm{hi}^{*}$ & 3.03 r.w & 15.2 o.r & 6.5 uvw & 0.448 uvw & $\begin{array}{l}6.4 \\
\text { u.x } \\
\end{array}$ \\
\hline & & $\mathrm{H}$ & $61.65 \mathrm{~d} . \mathrm{g}$ & $7.21 \mathrm{y}$ & $3.09 \mathrm{~m} . \mathrm{q}$ & $10.3 \mathrm{~s}$ & $711.4 \mathrm{y}$ & $2.28 \mathrm{yz}$ & $13.6 \mathrm{rst}$ & 8.27 k.o & 0.425 v.y & $\begin{array}{c}6.04 \\
\text { v.y }\end{array}$ \\
\hline & & S & $60.85 \mathrm{~d} . \mathrm{g}$ & $7.2 \mathrm{y}$ & 2.71 p.t & 9.91 stu & $220.2 \mathrm{u}^{*}$ & $12.8 \mathrm{a}$ & $25 \mathrm{~g}$ & $7.46 \mathrm{r}$ & $0.68 \mathrm{~g}$ & $\begin{array}{c}10.16 \\
\mathrm{~g}\end{array}$ \\
\hline & \multirow{3}{*}{ S5 } & $\mathrm{C}$ & $66.7 \mathrm{cde}$ & $6.15 \mathrm{de}^{*}$ & 2.11 t.w & $8.26 \mathrm{y}$ & $487.9 \mathrm{mn}^{*}$ & $5.46 \mathrm{~h}$ & $20.6 \mathrm{jk}$ & $5.14 \mathrm{zab}^{*}$ & $0.569 \mathrm{lmn}$ & $\begin{array}{c}8.31 \\
\text { j.o }\end{array}$ \\
\hline & & $\mathrm{H}$ & $67 \mathrm{cde}$ & $6.07 \mathrm{e}^{*}$ & 2.12 t.w & $8.19 \mathrm{y}$ & $589.6 \mathrm{~g}^{*}$ & 3.31 p.s & $19.5 \mathrm{kl}$ & $6.69 \mathrm{tu}$ & $0.559 \mathrm{mno}$ & 8.1 1.p \\
\hline & & s & 29.45 opq & $6.76 \mathrm{a}^{*}$ & 2.54 q.v & $9.3 \mathrm{vw}$ & $667.2 \mathrm{~b}^{*}$ & 3.6 nop & $28.9 \mathrm{e}$ & $7.05 \mathrm{~s}$ & $0.77 \mathrm{e}$ & $11.5 \mathrm{e}$ \\
\hline & \multirow{3}{*}{ S12 } & C & 42 i.p & $3.5 \mathrm{~m}^{*}$ & $1.02 \mathrm{yz}$ & $4.52 c^{*}$ & $388 \mathrm{~s}^{*}$ & $5.95 \mathrm{fg}$ & $21.7 \mathrm{ij}$ & $4.8 \mathrm{cde}^{*}$ & $0.592 \mathrm{klm}$ & $\begin{array}{c}8.67 \\
\mathrm{jkl}\end{array}$ \\
\hline & & $\mathrm{H}$ & 37.15 k.q & $4.41 \mathrm{k}^{*}$ & 2.27 r.w & $6.69 \mathrm{a}^{*}$ & $513 \mathrm{j}^{*}$ & $4.54 \mathrm{jk}$ & $27.8 \mathrm{ef}$ & $4.91 \mathrm{bcd}^{*}$ & $0.74 \mathrm{ef}$ & $\begin{array}{c}11.04 \\
\text { ef }\end{array}$ \\
\hline & & S & $33.3 \mathrm{m.q}$ & $5.69 \mathrm{~g}^{*}$ & $1.75 \mathrm{wx}$ & $7.43 \mathrm{z}$ & $442.1 \mathrm{p}^{*}$ & $4.97 \mathrm{i}$ & $90.3 \mathrm{a}$ & $5.34 \mathrm{yz}$ & $2.15 \mathrm{a}$ & $33.2 \mathrm{a}$ \\
\hline \multirow{12}{*}{ G2 } & \multirow{3}{*}{ S1 } & C & $26.7 \mathrm{pq}$ & $8.67 \mathrm{n}$ & $0.93 \mathrm{yz}$ & $9.6 \mathrm{uv}$ & $589.7 \mathrm{~g}^{*}$ & $1.91 \mathrm{z}$ & $15.4 \mathrm{opq}$ & $7.89 \mathrm{pq}$ & 0.451 t.w & $\begin{array}{l}6.45 \\
\text { uvw }\end{array}$ \\
\hline & & $\mathrm{H}$ & $55.35 \mathrm{f.i}$ & $22.89 \mathrm{a}$ & $8.76 \mathrm{~b}$ & $31.65 \mathrm{a}$ & $2116.6 \mathrm{a}$ & $2.19 \mathrm{yz}$ & 15.2 o.r & $10 \mathrm{c}$ & 0.461 s.v & $\begin{array}{l}6.6 \\
\text { tuv }\end{array}$ \\
\hline & & $S$ & 59.15 e.g & $10.46 \mathrm{~h}$ & 2.8 o.s & $13.27 \mathrm{jk}$ & $812.9 \mathrm{~s}$ & $2.27 \mathrm{yz}$ & 16.8 no & $9.28 \mathrm{e}$ & $0.497 \mathrm{pqr}$ & $\begin{array}{c}7.28 \\
\mathrm{rs}\end{array}$ \\
\hline & \multirow{3}{*}{ S2 } & C & 65.8 c.f & $7.91 \mathrm{v}$ & $0.25 \mathrm{a}^{*}$ & $7.49 \mathrm{z}$ & 483.8 no* $^{*}$ & $2.39 \mathrm{xyz}$ & $21.2 \mathrm{ij}$ & $5.06 \mathrm{ab}^{*}$ & $0.582 \mathrm{~lm}$ & $\begin{array}{l}8.51 \\
j . \mathrm{m} \\
\end{array}$ \\
\hline & & $\mathrm{H}$ & $53.3 \mathrm{f} . \mathrm{k}$ & 9.131 & $3.41 \mathrm{j} . \mathrm{n}$ & $12.54 \mathrm{~lm}$ & $924 n$ & $2.38 \mathrm{xyz}$ & $15.4 \mathrm{opq}$ & $7.98 \mathrm{opq}$ & 0.464 stu & $\begin{array}{c}6.66 \\
\mathrm{tu}\end{array}$ \\
\hline & & $S$ & 59.45 e.g & $8.93 \mathrm{~m}$ & $3.06 \mathrm{~m} \cdot \mathrm{q}$ & 11.99 no & $810.9 \mathrm{~s}$ & $2.45 \mathrm{xyz}$ & $17.4 \mathrm{mn}$ & $8.64 \mathrm{hi}$ & $0.514 \mathrm{pq}$ & $\begin{array}{l}7.48 \\
\mathrm{qrs}\end{array}$ \\
\hline & \multirow{3}{*}{ S5 } & C & 37.8 j.q & $6.17 \mathrm{~d}^{*}$ & $0.25 \mathrm{a}^{*}$ & $5.88 \mathrm{~b}^{*}$ & $391.4 \mathrm{~s}^{*}$ & $2.64 \mathrm{w} . \mathrm{z}$ & $23.4 \mathrm{gh}$ & $5.05 \mathrm{abc}^{*}$ & $0.63 \mathrm{hij}$ & $\begin{array}{c}9.29 \\
\text { hi }\end{array}$ \\
\hline & & $\mathrm{H}$ & 49.65 g.m & $8.84 \mathrm{~m}$ & $3.43 \mathrm{j} . \mathrm{n}$ & $12.27 \mathrm{mn}$ & $860 \mathrm{q}$ & 2.8 u.z & $17.6 \mathrm{mn}$ & $6.87 \mathrm{st}$ & $0.524 \mathrm{opq}$ & $\begin{array}{c}7.43 \\
\text { qrs }\end{array}$ \\
\hline & & $S$ & 49.15 g.m & $8.44 \mathrm{p}$ & 3.15 k.p & $11.6 \mathrm{op}$ & $791.4 \mathrm{t}$ & $2.61 \mathrm{w} . \mathrm{z}$ & $22.5 \mathrm{hi}$ & 8.23 k.o & $0.62 \mathrm{ijk}$ & $\begin{array}{c}9.29 \\
\text { hi }\end{array}$ \\
\hline & \multirow{6}{*}{ S12 } & $\mathrm{C}$ & 36 l.q & $3.77 \mathrm{l}^{*}$ & $0.27 \mathrm{a}^{*}$ & $4.05 \mathrm{~d}^{*}$ & $210 \mathrm{v}^{*}$ & 2.96 r.y & $24.6 \mathrm{~g}$ & $4.56 \mathrm{e}^{*}$ & 0.65 ghi & $9.7 \mathrm{gh}$ \\
\hline & & $\mathrm{H}$ & 37.05 k.q & $5.6 \mathrm{~h}^{*}$ & $17.44 \mathrm{a}$ & $23.04 \mathrm{~d}$ & $658.1 \mathrm{c}^{*}$ & $3.22 \mathrm{p} . \mathrm{t}$ & $20.5 \mathrm{jk}$ & $6.67 \mathrm{tu}$ & $0.57 \mathrm{ml}$ & $\begin{array}{c}8.46 \\
\text { j.n }\end{array}$ \\
\hline & & S & $25.35 \mathrm{pq}$ & $8.34 \mathrm{q}$ & $1.31 \mathrm{xy}$ & $9.64 \mathrm{uv}$ & $736.6 \mathrm{w}$ & 3.74 no & $26.5 \mathrm{f}$ & 6.59 uv & $0.71 \mathrm{f}$ & $\begin{array}{c}10.68 \\
\mathrm{f}\end{array}$ \\
\hline \multirow{12}{*}{ G3 } & & C & $28 \mathrm{opq}$ & $4.45 \mathrm{k}^{*}$ & 2.25 s.w & $6.7 \mathrm{a}^{*}$ & $489.3 \mathrm{~lm}^{*}$ & $2.64 \mathrm{w} . \mathrm{z}$ & $10.3 \mathrm{xy}$ & $7.86 \mathrm{q}$ & $0.337 \mathrm{~d}^{*}$ & $\begin{array}{l}4.64 \\
\mathrm{de}^{*}\end{array}$ \\
\hline & & $\mathrm{H}$ & $50.45 \mathrm{~g} .1$ & $6.81 \mathrm{a}^{*}$ & 2.25 s.w & $9.06 \mathrm{wx}$ & $585.6 \mathrm{gh}^{*}$ & $2.56 \mathrm{xyz}$ & 11.2 v.y & 8.6 hij & 0.369 a.d* & $\begin{array}{l}5.16 \\
\text { a.d } \\
\end{array}$ \\
\hline & & $S$ & $83.75 \mathrm{ab}$ & $9.64 \mathrm{k}$ & 3.31 j.o & $12.95 \mathrm{kl}$ & $757.9 \mathrm{v}$ & 2.78 u.z & 14.5 qrs & $9.06 \mathrm{ef}$ & $0.445 \mathrm{uvw}$ & $\begin{array}{l}6.45 \\
\text { uvw }\end{array}$ \\
\hline & \multirow{3}{*}{ S2 } & C & $51.5 \mathrm{~g} .1$ & $2.89 \mathrm{n}^{*}$ & $1.06 \mathrm{yz}$ & $3.94 \mathrm{~d}^{*}$ & $405 r^{*}$ & $2.72 \mathrm{v} . \mathrm{z}$ & 11.7 u.x & $6.4 \mathrm{uvw}$ & 0.369 a.d* & $\begin{array}{l}5.16 \\
\text { a.d }\end{array}$ \\
\hline & & $\mathrm{H}$ & $36.21 . q$ & $5.91 \mathrm{f}^{*}$ & 2.27 r.w & $8.19 \mathrm{y}$ & $580.1 \mathrm{i}^{*}$ & 2.86 t.y & 11.6 u.x & 8.27 k.o & 0.379 z.c ${ }^{*}$ & $\begin{array}{l}5.31 \\
\text { z.c. } \\
\end{array}$ \\
\hline & & S & $54.5 \mathrm{f} . \mathrm{k}$ & $7.34 \mathrm{x}$ & 2.64 p.u & $9.99 \mathrm{stu}$ & $681.7 \mathrm{z}$ & $3.06 \mathrm{r.v}$ & $17.4 \mathrm{mn}$ & $8.64 \mathrm{hi}$ & $0.51 \mathrm{pqr}$ & $\begin{array}{c}7.48 \\
\text { qrs }\end{array}$ \\
\hline & \multirow{3}{*}{ S5 } & C & $18.5 \mathrm{r}$ & $2.2 \mathrm{o}^{*}$ & $0.94 \mathrm{yz}$ & $3.14 \mathrm{e}^{*}$ & $301.8 \mathrm{t}^{*}$ & 2.96 r.y & 11.7 u.x & $4.76 \mathrm{ed}^{*}$ & 0.369 a.d* & $\begin{array}{l}5.16 \\
\text { a.d } \\
\end{array}$ \\
\hline & & $\mathrm{H}$ & $38.6 \mathrm{j} \cdot \mathrm{q}$ & $4.78 \mathrm{j}^{*}$ & 2.62 p.v & $7.41 \mathrm{z}$ & $493.1 \mathrm{kl}^{*}$ & 2.92 s.y & $14.5 \mathrm{qrs}$ & $6.55 \mathrm{uvw}$ & 0.445 uvw & $\begin{array}{l}6.35 \\
\text { u.x } \\
\end{array}$ \\
\hline & & $S$ & $55 \mathrm{f.j}$ & $5.89 \mathrm{f}^{*}$ & 2.64 p.u & $8.54 \mathrm{y}$ & $596 \mathrm{f}^{*}$ & 3.25 p.t & $21.4 \mathrm{ij}$ & $8.09 \mathrm{~m} . \mathrm{q}$ & $0.599 \mathrm{jkl}$ & $\begin{array}{c}8.88 \\
\text { ij } \\
\end{array}$ \\
\hline & \multirow{3}{*}{ S12 } & C & $38.5 \mathrm{j.q}$ & $1.03 \mathrm{p}^{*}$ & $0.71 \mathrm{yz}$ & $1.75 \mathrm{f}^{*}$ & $205.2 \mathrm{w}^{*}$ & $3.13 \mathrm{r.v}$ & $19.3 \mathrm{kl}$ & $2.1 \mathrm{f}^{*}$ & $0.556 \mathrm{mno}$ & $\begin{array}{c}7.84 \\
\text { o.r }\end{array}$ \\
\hline & & $\mathrm{H}$ & $32.85 \mathrm{n} . \mathrm{q}$ & $3.741^{*}$ & $0.65 \mathrm{z}$ & $4.38 \mathrm{~cd}^{*}$ & $479.4 \mathrm{o}^{*}$ & $3.58 \mathrm{n} \cdot \mathrm{q}$ & $77.7 \mathrm{~b}$ & $5.76 \mathrm{x}$ & $1.86 \mathrm{~b}$ & $\begin{array}{c}28.7 \\
\mathrm{~b}\end{array}$ \\
\hline & & $S$ & $21.3 \mathrm{q}$ & $5.51 \mathrm{i}^{*}$ & $2.03 \mathrm{vw}$ & $7.54 \mathrm{z}$ & $516 j^{*}$ & $3.8 \mathrm{mn}$ & $34.2 \mathrm{~d}$ & $5.44 \mathrm{y}$ & $0.88 \mathrm{~d}$ & $\begin{array}{c}13.4 \\
\mathrm{~cd}\end{array}$ \\
\hline \multirow{4}{*}{ G4 } & \multirow{3}{*}{ S1 } & C & 57.4 e.g & $6.38 \mathrm{bc}^{*}$ & $4.79 \mathrm{gh}$ & $11.17 \mathrm{pqr}$ & $610.9 \mathrm{~d}^{*}$ & $3.24 \mathrm{p} . \mathrm{t}$ & $9.9 \mathrm{y}$ & $7.41 \mathrm{r}$ & $0.333 \mathrm{~d}^{*}$ & $\begin{array}{c}4.54 \\
\mathrm{e}^{*}\end{array}$ \\
\hline & & $\mathrm{H}$ & $61.7 \mathrm{~d} . \mathrm{g}$ & $8.97 \mathrm{~m}$ & $8.1 \mathrm{c}$ & $17.07 \mathrm{~g}$ & $1347.9 \mathrm{~d}$ & $9.2 \mathrm{~b}$ & 11.9 u.x & $8.92 \mathrm{fg}$ & $0.349 \mathrm{~cd}^{*}$ & $\begin{array}{l}5.31 \\
\mathrm{z.c \textrm {c } ^ { * }} \\
\end{array}$ \\
\hline & & S & $83.15 \mathrm{ab}$ & $9.67 \mathrm{k}$ & $4.3 \mathrm{hi}$ & $13.97 \mathrm{i}$ & $1072.4 \mathrm{~g}$ & $8.23 \mathrm{c}$ & 12.8 utv & $9.57 \mathrm{~d}$ & 0.395 yza $^{*}$ & $\begin{array}{l}.62 \\
\mathrm{yza}^{*} \\
\end{array}$ \\
\hline & S2 & $\mathrm{C}$ & $50.55 \mathrm{~g} .1$ & $7.32 \mathrm{x}$ & $2.86 \mathrm{n} . \mathrm{r}$ & $10.18 \mathrm{st}$ & $683.7 \mathrm{z}$ & $4.41 \mathrm{kl}$ & 11.6 u.x & $6.89 \mathrm{st}$ & 0.366 a.d* & $\begin{array}{l}.16 \\
\text { a.d }\end{array}$ \\
\hline
\end{tabular}


Shokouhian A and Omidi H (2021). Not Bot Horti Agrobo 49(3):12063

\begin{tabular}{|c|c|c|c|c|c|c|c|c|c|c|c|c|}
\hline & & $\mathrm{H}$ & $80.05 \mathrm{abc}$ & $13.14 \mathrm{~d}$ & $5.94 \mathrm{f}$ & $19.09 \mathrm{e}$ & $1215.7 \mathrm{e}$ & $6.38 \mathrm{e}$ & $13.6 \mathrm{rst}$ & $8.49 \mathrm{ijk}$ & $0.405 \mathrm{x.a}^{*}$ & $\begin{array}{c}5.83 \\
\text { xyz }\end{array}$ \\
\hline & & S & $90.45 \mathrm{a}$ & $18.23 \mathrm{c}$ & $7.54 \mathrm{~d}$ & $25.77 \mathrm{c}$ & $1683.9 \mathrm{c}$ & $6.82 \mathrm{~d}$ & $14.7 \mathrm{qrs}$ & $10.1 \mathrm{c}$ & 0.474 r.u & $\begin{array}{c}6.35 \\
\text { u.x }\end{array}$ \\
\hline & \multirow{3}{*}{ S5 } & C & 45.45 h.o & $5.65 \mathrm{gh}^{*}$ & $2.89 \mathrm{n.q}$ & $8.53 \mathrm{y}$ & $417.2 \mathrm{q}^{*}$ & 3.03 r.w & $15.2 \mathrm{o.r}$ & $6.29 \mathrm{w}$ & 0.445 uvw & $\begin{array}{l}6.45 \\
\text { uvw }\end{array}$ \\
\hline & & $\mathrm{H}$ & 66.45 c.f & $12.15 \mathrm{e}$ & $5.13 \mathrm{~g}$ & $17.28 \mathrm{~g}$ & $1191.4 \mathrm{f}$ & $4.76 \mathrm{ijk}$ & $20.1 \mathrm{jkl}$ & $8.4 \mathrm{i} . \mathrm{l}$ & $0.579 \mathrm{~lm}$ & $\begin{array}{c}8.26 \\
\text { k.o } \\
\end{array}$ \\
\hline & & S & $85.7 \mathrm{a}$ & $11.51 \mathrm{~g}$ & 3.39 j.o & $14.9 \mathrm{~h}$ & $1045.7 \mathrm{i}$ & 3.36 o.r & $23.8 \mathrm{gh}$ & 8.17 l.p & $0.66 \mathrm{gh}$ & $\begin{array}{c}9.24 \\
\mathrm{hi} \\
\end{array}$ \\
\hline & \multirow{3}{*}{ S12 } & $\mathrm{C}$ & 38.55 j.q & $3.52 \mathrm{~m}^{*}$ & 3.12 l.q & $6.64 \mathrm{a}^{*}$ & $298 \mathrm{t}^{*}$ & $2.31 \mathrm{xyz}$ & $20.3 \mathrm{jk}$ & $5.18 \mathrm{yza}^{*}$ & $0.569 \mathrm{lmn}$ & $8 \mathrm{~m} \cdot \mathrm{q}$ \\
\hline & & $\mathrm{H}$ & $65.2 \mathrm{c.f}$ & 8.06 tu & $2.04 \mathrm{uvw}$ & $10.1 \mathrm{st}$ & $779.7 \mathrm{u}$ & 3.22 p.t & $24.3 \mathrm{~g}$ & $8.08 \mathrm{~m} . \mathrm{q}$ & $0.668 \mathrm{~g}$ & $\begin{array}{c}8.82 \\
\mathrm{ijk}\end{array}$ \\
\hline & & S & $76.1 \mathrm{bcd}$ & $9.77 \mathrm{j}$ & $3.84 \mathrm{ij}$ & $13.6 \mathrm{ij}$ & $894.3 \mathrm{p}$ & $3.02 \mathrm{r} . \mathrm{x}$ & $36.8 \mathrm{c}$ & $8.36 \mathrm{j.m}$ & $0.94 \mathrm{c}$ & $13.7 \mathrm{c}$ \\
\hline \multirow{12}{*}{ G5 } & \multirow{3}{*}{ S1 } & C & $91.12 \mathrm{a}$ & $8.49 \mathrm{o}$ & $3.53 \mathrm{j} . \mathrm{m}$ & 12.02 no & $604.1 \mathrm{e}^{*}$ & 3 r.x & 12.2 t.w & $12.35 \mathrm{a}$ & $0.356 \mathrm{bcd}^{*}$ & $\begin{array}{c}4.9 \\
\text { b. e* }\end{array}$ \\
\hline & & $\mathrm{H}$ & $84.3 \mathrm{a}$ & 9.181 & $3.67 \mathrm{jk}$ & $12.85 \mathrm{kl}$ & $814.2 \mathrm{~s}$ & 3.03 r.w & 11 wxy & $9.09 \mathrm{ef}$ & $0.353 \mathrm{~cd}^{*}$ & $\begin{array}{l}4.75 \\
\text { cde }^{*}\end{array}$ \\
\hline & & S & $87.05 \mathrm{a}$ & $20.05 \mathrm{~b}$ & $7.7 \mathrm{~cd}$ & $27.75 \mathrm{~b}$ & $495.8 \mathrm{k}^{*}$ & $3.02 \mathrm{r} . \mathrm{x}$ & 12.6 u.w & 8.141.p & 0.392 y.b* & $\begin{array}{l}5.47 \\
\text { y.b* }\end{array}$ \\
\hline & & $\mathrm{C}$ & $60.05 \mathrm{~d} . \mathrm{g}$ & $8.13 \mathrm{st}$ & 3.42 j.n & 11.55 op & 976.81 & $5.07 \mathrm{i}$ & $13.6 \mathrm{rst}$ & $11.7 \mathrm{~b}$ & 0.415 w.z & $\begin{array}{l}5.99 \\
\text { wxy } \\
\end{array}$ \\
\hline & S2 & $\mathrm{H}$ & $87.5 \mathrm{a}$ & $8.15 \mathrm{~s}$ & $3.74 \mathrm{jk}$ & 11.89 no & $830.6 \mathrm{r}$ & 3.19 q.u & 12.5 t.w & $8.32 \mathrm{k} . \mathrm{n}$ & 0.405 x.a* $^{*}$ & $\begin{array}{c}6.35 \\
\text { u.x } \\
\end{array}$ \\
\hline & & S & 74.65 bcd & $11.63 \mathrm{f}$ & $6.54 \mathrm{e}$ & $18.16 \mathrm{f}$ & $932.9 \mathrm{~m}$ & $3.53 \mathrm{n} . \mathrm{q}$ & $15.4 \mathrm{opq}$ & $7.33 \mathrm{r}$ & 0.468 stu & $\begin{array}{c}6.5 \\
\text { uvw }\end{array}$ \\
\hline & & $\mathrm{C}$ & $61.6 \mathrm{~d} . \mathrm{g}$ & $6.95 \mathrm{z}$ & $5.07 \mathrm{~g}$ & $12.02 \mathrm{no}$ & $987.4 \mathrm{k}$ & $5.71 \mathrm{gh}$ & $15 \mathrm{pqr}$ & $9.28 \mathrm{e}$ & 0.441 u.x & $\begin{array}{c}6.5 \\
\text { uvw }\end{array}$ \\
\hline & S5 & $\mathrm{H}$ & $80.9 \mathrm{abc}$ & $7.99 \mathrm{u}$ & 3.34 j.o & $11.33 \mathrm{pq}$ & 899.3 o & $4.8 \mathrm{ij}$ & 16.8 no & 8.19 l.o & $0.494 \mathrm{qrs}$ & $\begin{array}{l}7.64 \\
\text { p.s }\end{array}$ \\
\hline & & $S$ & $75.95 \mathrm{bcd}$ & $10.08 \mathrm{i}$ & $3.91 \mathrm{ij}$ & $13.99 \mathrm{i}$ & $1066.8 \mathrm{~h}$ & $5.98 \mathrm{fg}$ & $23.4 \mathrm{gh}$ & $6.52 \mathrm{uvw}$ & $0.628 \mathrm{j}$ & $\begin{array}{c}9.49 \\
\mathrm{~h}\end{array}$ \\
\hline & & $\mathrm{C}$ & $56.5 \mathrm{fgh}$ & $6.33 c^{*}$ & $2.05 \mathrm{uvw}$ & $8.38 \mathrm{y}$ & $997.3 \mathrm{j}$ & $6.23 \mathrm{ef}$ & $18.6 \mathrm{~lm}$ & $7.91 \mathrm{pq}$ & $0.54 \mathrm{npo}$ & $\begin{array}{c}7.89 \\
\text { n.q } \\
\end{array}$ \\
\hline & S12 & $\mathrm{H}$ & $60.35 \mathrm{~d} . \mathrm{g}$ & $7.78 \mathrm{w}$ & $3.02 \mathrm{~m} \cdot \mathrm{q}$ & $10.8 \mathrm{r}$ & $996.1 \mathrm{j}$ & $4.1 \mathrm{~lm}$ & $28.9 \mathrm{e}$ & $7.57 \mathrm{r}$ & $0.75 \mathrm{e}$ & $11.5 \mathrm{e}$ \\
\hline & & $S$ & 45.72 h.o & $6.46 b^{*}$ & 2.24 s.w & $8.69 x y$ & $1818.8 \mathrm{~b}$ & $7.92 \mathrm{c}$ & $33.5 \mathrm{~d}$ & $6.32 \mathrm{vw}$ & $0.86 \mathrm{~d}$ & $\begin{array}{c}13.1 \\
\mathrm{~d}\end{array}$ \\
\hline
\end{tabular}

Means followed by the same letter in each column are not significantly different according to Duncan test at $5 \%$ level Genotypes (G1: Shokofa; G2: Sina; G3: Paya; G4: Turbata; G5: Aria)

C: control (non-priming); H: Hyrdopriming; S: zinc-sulphate

\section{Correlation coefficients}

As shown in Table 5, GP was significantly and positively correlated with RG, seedling growth parameters, photosynthetic pigment contents, and protein content while negatively correlated with MGT, UG, SOD activity, proline, and sugar contents. The correlation results illustrated that the SOD activity and proline and sugar contents were negatively and significantly correlated with the pigment contents.

Table 5. Correlation coefficients among germination indices and physiological characteristics of sugar beet genotypes under salinity stress and priming treatments

\begin{tabular}{|c|c|c|c|c|c|c|c|c|c|c|c|c|c|c|c|c|c|}
\hline & GP & MGT & GR & UG & RL & PL & SL & SVI & RWC & $\mathrm{Chla}$ & $\mathrm{Chlb}$ & $\begin{array}{l}\text { Total } \\
\text { chl }\end{array}$ & Caro & Antho & SOD & Protein & Proline \\
\hline MGT & $-0.90^{* *}$ & & & & & & & & & & & & & & & & \\
\hline $\begin{array}{l}\text { GR } \\
\end{array}$ & $0.47^{* *}$ & $-0.59^{* *}$ & & & & & & & & & & & & & & & \\
\hline UG & $-0.91^{* *}$ & $0.86^{* *}$ & $-0.36^{*+}$ & & & & & & & & & & & & & & \\
\hline RL & $0.50^{* *}$ & $-0.40^{* *}$ & $0.16^{*}$ & $0.42^{* *}$ & & & & & & & & & & & & & \\
\hline PL & $0.43^{* 4}$ & $-0.41^{* *}$ & $0.37^{* *}$ & $0.44^{*}$ & $0.78^{* *}$ & & & & & & & & & & & & \\
\hline SL & $0.50^{* *}$ & $-0.43^{* *}$ & $0.36^{* *}$ & $0.4^{7 *}$ & $0.96^{* *}$ & $0.92^{* *}$ & & & & & & & & & & & \\
\hline SVI & $0.43^{* *}$ & $0.44^{* *}$ & $0.33^{* *}$ & $0.30^{* *}$ & $0.91^{* *}$ & $0.93^{* *}$ & $0.97^{* *}$ & & & & & & & & & & \\
\hline RWC & $0.11 \mathrm{~ns}$ & $-0.16 \mathrm{~ns}$ & $0.14 \mathrm{~ns}$ & $0.13 \mathrm{~ns}$ & $0.04 \mathrm{~ns}$ & $0.09 \mathrm{~ns}$ & $0.06 \mathrm{~ns}$ & $0.08 \mathrm{~ns}$ & & & & & & & & & \\
\hline $\begin{array}{l}\mathrm{Chla} \\
\end{array}$ & $0.71^{* *}$ & $-0.65^{* *}$ & $0.35^{* *}$ & $0.69^{* *}$ & $0.35^{* *}$ & $0.35^{* *}$ & $0.37^{* *}$ & $0.34^{* *}$ & $0.19 \mathrm{~ns}$ & & & & & & & & \\
\hline Chlb & $0.35^{*}$ & $-0.34^{* *}$ & $0.21^{*}$ & $0.30^{*}$ & $0.25^{*}$ & $0.22^{*}$ & $0.25^{*}$ & $0.24^{*}$ & 0.08 & $0.49^{* *}$ & & & & & & & \\
\hline $\begin{array}{c}\text { Total } \\
\mathrm{chl} \\
\end{array}$ & $0.64^{* *}$ & $-0.60^{* *}$ & $0.34^{* *}$ & $0.56^{* *}$ & $0.36^{* *}$ & $0.35^{* *}$ & $0.37^{* *}$ & $0.34^{* *}$ & $0.21^{*}$ & $0.91^{* *}$ & $0.81^{* *}$ & & & & & & \\
\hline Caro & $0.53^{* *}$ & $-0.52^{* *}$ & $0.30^{* *}$ & $0.40^{* *}$ & $0.20 \mathrm{~ns}$ & $0.23^{*}$ & $0.22^{*}$ & $0.21^{*}$ & 0.07 & $0.69^{* *}$ & $0.45^{5 *}$ & $0.68^{* *}$ & & & & & \\
\hline Antho & $0.04 \mathrm{~ns}$ & $0.12 \mathrm{~ns}$ & $0.05 \mathrm{~ns}$ & $0.01 \mathrm{~ns}$ & $-0.01 \mathrm{~ns}$ & $0.11 \mathrm{~ns}$ & $0.03 \mathrm{~ns}$ & $0.08 \mathrm{~ns}$ & $0.01 \mathrm{~ns}$ & $0.03 \mathrm{~ns}$ & $0.07 \mathrm{~ns}$ & $0.06 \mathrm{~ns}$ & $0.1 \mathrm{~ns}$ & & & & \\
\hline SOD & $-0.28^{*}$ & $0.34^{* *}$ & $-0.60^{* *}$ & $0.20 \mathrm{~ns}$ & $-0.14 \mathrm{~ns}$ & $-0.21^{*}$ & $-0.18 \mathrm{~ns}$ & $-0.18 \mathrm{~ns}$ & $-0.12 \mathrm{~ns}$ & $-0.20 \mathrm{~ns}$ & $-0.19 \mathrm{~ns}$ & $-0.21^{*}$ & $-0.20 \mathrm{~ns}$ & $0.10 \mathrm{~ns}$ & & & \\
\hline $\begin{array}{l}\text { Protein } \\
\end{array}$ & $0.50^{* *}$ & $-0.54^{* *}$ & $0.38^{* *}$ & $0.12 \mathrm{~ns}$ & $0.40^{* *}$ & $0.41^{* *}$ & $0.43^{* *}$ & $0.42^{2 *}$ & $0.37^{* *}$ & $0.58^{* *}$ & $0.37^{7^{*}}$ & $0.56^{* \mathrm{*}}$ & $0.54^{* *}$ & $0.03 \mathrm{~ns}$ & $-0.40^{* *}$ & & \\
\hline $\begin{array}{l}\text { Proline } \\
\end{array}$ & $-0.28^{*}$ & $0.34^{* *}$ & $-0.60^{* 4}$ & $0.22^{*}$ & $-0.12 \mathrm{~ns}$ & $-0.19 \mathrm{~ns}$ & $-0.16 \mathrm{~ns}$ & $\begin{array}{ll}-0.16 \mathrm{~ns} \\
\end{array}$ & $\begin{array}{ll}-0.13 \mathrm{~ns} \\
\end{array}$ & $-0.2 \mathrm{~ns}$ & $\begin{array}{l}-0.2 \mathrm{~ns} \\
\end{array}$ & $-0.22^{*}$ & $\begin{array}{l}-0.1 \text { ns } \\
\end{array}$ & $0.09 \mathrm{~ns}$ & $0.99^{*}$ & $\begin{array}{l}-0.34^{*} \\
\end{array}$ & \\
\hline Sugar & $-0.27^{*}$ & $0.33^{* *}$ & $-0.60^{*+}$ & $0.22^{*}$ & $-0.12 \mathrm{~ns}$ & $-0.19 \mathrm{~ns}$ & $-0.15 \mathrm{~ns}$ & $-0.16 \mathrm{~ns}$ & -0.13 & $-0.2 \mathrm{~ns}$ & $-0.2 \mathrm{~ns}$ & $-0.22^{*}$ & $-0.1 \mathrm{~ns}$ & $0 . \operatorname{lns}$ & $0.99^{* *}$ & $-0.34^{*}$ & $0.99^{* *}$ \\
\hline
\end{tabular}

ns: non-significant; ${ }^{*}$ and ${ }^{* *}$ : significant at 5 and $1 \%$ probability levels 


\section{Discussion}

The aims of this study were to (i) characterize the germination and physiological response to different salinity stress, hydropriming and osmopriming $\left(\mathrm{ZnSO}_{4}\right)$ and (ii) investigate whether hydropriming and osmopriming induce tolerance to salinity in seeds of sugar beet genotypes. Results indicated that the salinity stress and priming significantly affected germination indices (GP, MGT, GR, SL, PL, RL, and SVI) and physiological attributes (RWC, photosynthetic pigments, anthocyanin, SOD activity, protein, and proline contents. As shown in our results, germination indices including GP, GR, RL, PL, SL, and SVI decreased and increased in sugar beet genotypes submitted to salinity and priming treatment, respectively, compared to the control. Gheidary et al. (2017) reported that the salinity can also affect different stages of plant life such as germination, length of root, and stem because as salinity increases water absorption by the seed is decreased which shows the inhibitory effect of salinity on seed germination. More other researchers also reported the deleterious effects of salinity $(\mathrm{NaCl})$ on seed germinability and seedling growth of many crops (Aghighi Shahverdi et al., 2017). The reasons for reduced germination percentage can be attributed to primary water uptake reduction and the adverse effect of osmotic potential and ion toxicity on physiological processes of seed germination (Javadi et al., 2016; Aghighi Shahverdi et al., 2017). Notable point a small increase in $\mathrm{NaCl}$ concentration in comparison to the control (non-stress), caused increase seed germination percentage. Aghighi Shahverdi et al. (2017) founded the reason for this may be due to the stimulatory effect of sodium chloride, which has the effect of stimulating out of root in seed and increases the germination percentage at low concentration of salt. In this regard, reported that seed priming more efficient to improve the performance of cucumber seedling in bio-saline water (Matias et al., 2015).

Seed priming has proved to be an effective method in imparting stress tolerance to plants using natural and/or synthetic compounds to treat the seeds before germination (Moreno et al., 2018). Different priming techniques have been used to enhance salinity tolerance in several species and several priming agents have proved to be effective. Therefore, no standard methods to treat seeds or target osmotic or ionic effects of salt stress are clearly defined. For example, improved germination parameters in salinity stress resulted from osmopriming seeds with solutions of low water potential for Amaranthus, while for quinoa, this effect was achieved from hydropriming and osmopriming seeds with solutions of high-water potential (Moreno et al., 2018). In the present study, hydropriming had the highest germination indices among different seed priming treatments. Seed hydropriming and osmopriming caused significant improvements in GU, reflected in high GP, high GR and reduced MGT under salinity. The superiority of hydropriming as a treatment in increasing plant germination indices compared to other treatments may be due to the effect of the absorption of more water by seeds compared to other treatments, they reached the highest percentage of germination as was reported by Gheidary et al. (2017). Similar results were reported by Kaur et al. (2002) who found applying hydropriming on seeds increases $\mathrm{RL}$ and stem the growth of pea plant. It was reported that $\mathrm{ZnSO}_{4}$ seed priming treatments may be useful tools due to their positive effects on GR and GP of lentil. Moreno et al. (2018) reported that the primed tolerance to moderate salinity was achieved for Chenopodium quinoa and Amaranthus caudatus species, the salinity threshold for germination to occur was slightly broadened. Finally, seed priming has two beneficial effects, firstly caused will increase germination indices such as GP, GR, seedling growth parameters, and SVI and secondly, moderate the adverse effects of salinity stress due to $\mathrm{NaCl}$, that between priming treatments, hydropriming had more positive effects on germination indices.

Seed priming has been suggested as one of the most useful physiological approaches to adapt glycophyte species to saline conditions. Pavia et al. (2019) concluded that the $\mathrm{Zn}$ treatments lessened the non-regulated energy dissipation caused by environmental stress, protected the plants against irreversible damages to the photosynthetic apparatus and enabled a better recovery of wheat plants after stress relief. The well-known effect from priming regarding faster, higher, and uniform germination was observed under optimal and suboptimal salinity conditions, which represent an overall improvement in seed vigor (Moreno et al., 2018). Aghighi Shahverdi et al. (2017) reported that the priming enhanced seed performances are related to the repair and the 
build-up of nucleic acid, enhanced synthesis of protein, repair of membranes and improves the antioxidant system. Mahmood et al. (2019) illustrated that increased levels of stress caused a decrease in GP, GR, and SVI of all chickpea cultivars while $\mathrm{ZnSO}_{4}$ seed priming treatment enhances the values of all these attributes under all drought levels. Increasing SVI as a result of priming with $\mathrm{ZnSO}_{4}$ or distilled water can result in a significant increase in SL and GP (the two parameters involved in the calculation of SVI), that maybe are because of the role of these treatments in cell division and cell elongation or cell division and growth meristematic (Noor-UnNisa Memon et al., 2013; Aghighi Shahverdi et al., 2017).

This study revealed that seedling growth from $\mathrm{ZnSO}_{4}$ primed seeds showed an increase in values of all germination attributes. According to previous research, it has been reported that in wheat plants $\mathrm{Zn}$ plays an effective role in the production of increased dry weight (Mahmood et al., 2019). While having comparison with results provided on seed priming effect on growth parameters of maize, it has been revealed that $\mathrm{ZnSO}_{4}$ seed priming treatment influenced positively shoot and root length of maize and pulses (Ambika and Balakrishnan, 2015). An increased root length of plants grown from seeds primed with $\mathrm{ZnSO}_{4}$ as compared to unprimed seeds could be the result of extensibility in the cell wall of the embryo (Mahmood et al., 2019). Also, the same researcher reported that seed priming with $\mathrm{ZnSO}_{4}$ solution decreased the resistance mechanism of endosperm envelope against growth permitting turgor threshold for germination as compared to non-primed seeds resulting increased shoot and root length (Mahmood et al., 2019).

In the present study, salinity caused reduces of chlorophyll contents (Table 3). While seed priming led to an increase in the mean of photosynthetic pigments under control and salinity stress conditions (Table 4). It seems that one of the reasons for the good tolerance of sugar beet, in the germination stage, to salinity stress is to moderate the negative effects of stress using priming treatments. Increasing the amount of photosynthetic pigments thus seed priming is stated in different reports (Noor-Un-Nisa Memon et al., 2013; Aghighi Shahverdi and Omidi 2015; Aghighi Shahverdi et al., 2017). The reduction of photosynthesis pigment is one of the major consequences of salinity or drought stresses in plants. This decrease may be due to stomatal or nonstomata limitations (Pavia et al., 2019). Stomatal closure is usually considered the determining factor in the reduction of $\mathrm{CO} 2$ assimilation and photosynthesis reduction during environmental stress such as salinity or drought (Abid et al., 2016). Nonetheless, , non-stomatal limitations may also occur at moderate and severe drought events (Brito et al., 2018). In our study, reduction of photosynthesis pigment contents such as chlorophyll a, b, total as well as carotenoids were observed by increasing $\mathrm{NaCl}$ concentration (Table 3). Generally, Mosavikia et al. (2020) concluded that limited stomatal conductance, diminished activities of carbon fixation enzymes, reduced quantities of photosynthetic pigments, and destruction of photosynthetic apparatus are among the key factors limiting the process of photosynthesis under salinity stress conditions.

On the other hand, the results of the current experiment showed that all sugar beet genotypes primed with $\mathrm{ZnSO}_{4}$ gave higher values of all chlorophyll pigment (chlorophyll a and total chlorophyll) as compared to unprimed seeds. This increase may be due to $\mathrm{Zn}$ which functions as a structural, functional, and catalytic component of enzymes, proteins and also a co-factor for normal growth and development of biosynthetic pigments (Samreen et al., 2017). The results presented are the agreement with the findings of Mahmood et al. (2019). The absorption of excess energy in the photosynthetic apparatus may be the main cause of ROS in large concentrations it could be escaped by degrading the pigments responsible for their absorption (Mahmood et al., 2019).

In the current study, the higher accumulation of carotenoid in 'Sina' sugar beet genotype cultivars under non-stress conditions could result in a positive effect on germination and seedling growth by affecting RWC and chlorophyll content as reported by Talebi et al. (2013). Carotenoids have a critical role as photoprotective compounds by quenching triplet and singlet oxygen derived from excess light energy, thus limiting membrane damage (Mahmood et al., 2019). However, the more enhanced increase in chlorophyll and carotenoid content in 'Sina' sugar beet genotype raised from seeds primed with distilled water as compared to unprimed seeds under control conditions. 
Enzymatic and non-enzymatic antioxidants response are defensive mechanisms to overcome oxidative stress (Demidchik, 2015). Proline and sugar, non-enzymatic antioxidants response, are known to act as an osmolyte/osmoprotectant agent under drought or salinity stress (Mosavikia et al., 2020). The osmolyte has an important role in scavenging free radicals, osmotic pressure adjustment, stabilizing sub-cellular structures, and storing carbon and nitrogen (Gorzi et al., 2017). The results of the current study concluded that a significant increase in the free proline and sugar contents as well as the activity of SOD enzyme under salt stress especially in $12 \mathrm{dS} / \mathrm{m} \mathrm{NaCl}$ level. Salinity stress in this experiment caused reduces of photosynthetic pigment contents and protein content while increased of SOD activity, proline and sugar contents. However priming treatment, by increases means of these traits causing moderated the adverse effect of salinity stress on biochemical characteristics of the sugar beet seedling, so that reduction of the amount of chlorophyll was minor in terms of priming treatments in high salinity levels (Table 3). Proline acts as a signaling/regulatory molecule and in the case of salt stress will be able to enhance the resistance of the plant to salinity (Javadi et al., 2016). A positive relationship between proline accumulation and the antioxidant level in the plant that the ROS caused by stress increases and leads to the accumulation of free proline in the plant reported by Zhang et al. (2006) which is in line with the findings of the correlation between traits in the present study. Aghighi Shahverdi et al. (2017) reported that the seed priming by increasing the activity of the enzyme proline 5-carboxylase synthase, as a key enzyme in the synthesis of proline increased synthesis of these secondary metabolites in the high salinity stress conditions.

Wang et al. (2013) reported that the antioxidant activity of SOD increased under salinity stress that the answer is crop tolerance to salinity conditions. Similar results were obtained in the present study. Salinity increases the activity of antioxidant enzymes were all priming treatments. Ambika and Balakrishnan (2015) reported that seed priming treatment caused an increase in the activity of ROS scavenging enzymes to enhance plant strength and viability. Metabolic activities are expected to increase remarkably in seeds following their priming which may lead to the higher activity of ROS as secondary products of mitochondrial respiration. There is strong evidence that antioxidant enzymes and free radicals are abundantly produced within seeds during germination, and are cooperatively tackled by enzymatic reactions (Noor-Un-Nisa Memon et al., 2013; Aghighi Shahverdi et al., 2017). The enhanced expression and activity of antioxidant enzymes as recorded in our studies have been proposed as part of seed strategy to cope with ROS produced during seed priming (Chiu et al., 2005). Hydropriming is the simplest approach to hydrate seeds and minimize the use of chemicals. However, if the seeds are not accurately hydrated, the hydration rate cannot be exactly controlled. It was observed that hydropriming practically ensured rapid and uniform germination accompanied by low abnormal seedling percentage.

\section{Conclusions}

Salinity $(\mathrm{NaCl})$ stress harmed seed germination and seedling growth of sugar beet genotypes. While seed priming (especially hydropriming) under high salinity level was caused by highly improved germination parameters and reduced germination time to promote early seed germination and harmonized growth. Also, seed priming with $\mathrm{ZnSO}_{4}$ and distilled water has improved germination indices and seedling physiological parameters such as photosynthetic pigments, proline, sugar, anthocyanin, and the activity of antioxidant enzyme. Generally, the most pronounced effect of seed priming was related to hydropriming and 'Aria' sugar beet genotype. The finding of this study leads to the conclusion that seed priming with $\mathrm{ZnSO}_{4}$ and distilled water by improving physiological mechanisms such as synthesis of photosynthetic pigments, antioxidant enzyme activity, proline amino acid, soluble sugar, carotenoids and anthocyanin moderated the negative effects of high salinity stress and increased GP, GR, and seedling growth parameters such as RL, PL, SL, and SVI. Also, genotypes 'Paya' and 'Aria' showed high tolerance and genotype 'Shokofa' showed low tolerance to salinity stress at the germination stage. 


\section{Authors' Contributions}

Both authors read and approved the final manuscript.

\section{Acknowledgements}

This research received no specific grant from any funding agency in the public, commercial, or not-forprofit sectors.

\section{Conflict of Interests}

The authors declare that there are no conflicts of interest related to this article.

\section{References}

Abid M, Tian Z, Ata-Ul-Karim S T, Wang F, Liu Y, Zahoor R, Jiang D, Dai T (2016). Adaptation to and recovery from drought stress at vegetative stages in wheat (Triticum aestivum) cultivars. Functional Plant Biology 43:1159-1169. https://doi.org/10.1071/FP16150

Aghighi Shahverdi M, Omidi H (2016). Effect of hormone priming and hydro priming on Stevia (Stevia rebaudiana Bertoni) seed germination under salt stress. Iranian Journal of Seed Sciences and Research 3(2):97-108.

Aghighi Shahverdi M, Omidi H, Tabatabaei SJ (2017). Effect of nutri-priming on germination indices and physiological characteristics of stevia seedling under salinity stress. Journal of Seed Science 39:353-362. http://dx.doi.org/10.1590/2317-1545v39n4172539

Ambika S, Balakrishnan K (2015). Enhancing germination and seedling vigour in cluster bean by organic priming. Scientific Research and Essays 10:298-301. https://doi.org/10.5897/SRE2015.6197

Anwar A, Xianchang Y, Yansu L (2020). Seed priming as a promising technique to improve growth, chlorophyll, photosynthesis and nutrient contents in cucumber seedlings. Notulae Botanicae Horti Agrobotanici Cluj-Napoca 48:116-127. https://doi.org/10.15835/nbha48111806

Bates LS, Waldren RP, Teare I (1973). Rapid determination of free proline for water-stress studies. Plant and Soil 39:205207. https://doi.org/10.1007/BF00018060

Beauchamp C, Fridovich I (1971). Superoxide dismutase: improved assays and an assay applicable to acrylamide gels. Analytical Biochemistry 44:276-287. https://doi.org/10.1016/0003-2697(71)90370-8

Bradford MM (1976). A rapid and sensitive method for the quantitation of microgram quantities of protein utilizing the principle of protein-dye binding. Analytical Biochemistry 72:248-254. https://doi.org/10.1016/00032697(76)90527-3

Brito C, Dinis L-T, Meijón M, Ferreira H, Pinto G, Moutinho-Pereira J, Correia C (2018). Salicylic acid modulates olive tree physiological and growth responses to drought and rewatering events in a dose dependent manner. Journal of Plant Physiology 230:21-32. https://doi.org/10.1016/j.jplph.2018.08.004

Carvalho RF, Piotto FA, Schmidt D, Peters LP, Monteiro CC, Azevedo RA (2011). Seed priming with hormones does not alleviate induced oxidative stress in maize seedlings subjected to salt stress. Scientia Agricola 68:598-602. https://doi.org/10.1590/S0103-90162011000500014

Chiu K, Chen C, Sung J (2005). Why low temperature primed sh-2 sweet corn seeds have better storability: some physiological clues. Seed Science and Technology 33:199-213. https://doi.org/10.15258/sst.2005.33.1.20

Demidchik V (2015). Mechanisms of oxidative stress in plants: from classical chemistry to cell biology. Environmental and Experimental Botany 109:212-228. https://doi.org/10.1016/j.envexpbot.2014.06.021 
Feghhenabi F, Hadi H, Khodaverdiloo H, van Genuchten M T (2020). Seed priming alleviated salinity stress during germination and emergence of wheat (Triticum aestivum L.). Agricultural Water Management 231:106022. https://doi.org/10.1016/j.agwat.2020.106022

Gheidary S, Akhzari D, Pessarakli M (2017). Effects of salinity, drought, and priming treatments on seed germination and growth parameters of Lathyrus sativus L. Journal of Plant Nutrition 40:1507-1514. https://doi.org/10.1080/01904167.2016.1269349

Gorzi A, Omidi H, Bostani A (2017). Morpho-physiological responses of Stevia (Stevia rebaudiana Bertoni) to various priming treatments under drought stress. Applied Ecology and Environmental Research 16:4753-4771. https://doi.org/10.15666/aeer/1604_47534771

Javadi A, Khomari S, Sofalian O (2016). Seed vigor and boron and calcium nutrition influence oilseed rape germinability and seedling growth under salt stress. Journal of Plant Nutrition 39:1688-1696. https://doi.org/10.1080/01904167.2015.1093138

Kaur S, Gupta A K, Kaur N (2002). Effect of osmo-and hydropriming of chickpea seeds on seedling growth and carbohydrate metabolism under water deficit stress. Plant Growth Regulation 37:17-22. https://doi.org/10.1023/A:1020310008830

Khaing M, Ultra Jr V, Chul Lee S (2020). Seed priming influence on growth, yield, and grain biochemical composition of two wheat cultivars. Journal of Agricultural Science and Technology 22:875-888.

Lichtenthaler HK, Buschmann C (2001). Chlorophylls and carotenoids: Measurement and characterization by UV-VIS spectroscopy. Current Protocols in Food Analytical Chemistry 1(1):F4-3.

Lv X, Chen S, Wang Y (2019). Advances in understanding the physiological and molecular responses of sugar beet to salt stress. Frontiers in Plant Science 10:1431. https://doi.org/10.3389/fpls.2019.01431

Mahmood A, Kanwal H, Kausar A, Ilyas A, Akhter N, Ilyas M, Nisa Z, Khalid H (2019). Seed priming with zinc modulate growth, pigments and yield of chickpea (Cicer arietinum L.) under water deficit conditions. Applied Ecology and Environmental Research 17:147-160. https://doi.org/10.15666/aeer/1701_147160

Mancinelli AL (1990). Interaction between light quality and light quantity in the photoregulation of anthocyanin production. Plant Physiology 92:1191-1195. Https://doi.org/10.1104/pp.92.4.1191

Matias JR, Ribeiro RC, Aragão CA, Araújo GGL, Dantas BF (2015). Physiological changes in osmo and hydroprimed cucumber seeds germinated in biosaline water. Journal of Seed Science 37:07-15. https://doi.org/10.1590/23171545 v37n1135472

Moreno C, Seal C, Papenbrock J (2018). Seed priming improves germination in saline conditions for Chenopodium quinoa and Amaranthus caudatus. Journal of Agronomy and Crop Science 204:40-48. https://doi.org/10.1111/jac. 12242

Mosavikia AA, Mosavi SG, Seghatoleslami M, Baradaran R (2020). Chitosan nanoparticle and pyridoxine seed priming improves tolerance to salinity in milk thistle seedling. Notulae Botanicae Horti Agrobotanici Cluj-Napoca 48:221233. https://doi.org/10.15835/nbha48111777

Noor-Un-Nisa MEMONGM, Pahoja V, Sharif N (2013). Response of seed priming with boron on germination and seedling sprouts of broccoli. International Journal of Agricultural Science and Research (IJASR) 1:163-174.

Paparella S, Araújo S, Rossi G, Wijayasinghe M, Carbonera D, Balestrazzi A (2015). Seed priming: state of the art and new perspectives. Plant Cell Reports 34:1281-1293. https://doi.org/10.1007/s00299-015-1784-y

Pavia I, Roque J, Rocha L, Ferreira H, Castro C, Carvalho A, Silva E, Brito C, Gonçalves A, Lima-Brito J (2019). Zinc priming and foliar application enhances photoprotection mechanisms in drought-stressed wheat plants during anthesis. Plant Physiology and Biochemistry 140:27-42. https://doi.org/10.1016/j.plaphy.2019.04.028

Rehman A, Farooq M, Naveed M, Nawaz A, Shahzad B (2018). Seed priming of Zn with endophytic bacteria improves the productivity and grain biofortification of bread wheat. European Journal of Agronomy 94:98-107. https://doi.org/10.1016/j.eja.2018.01.017

Saadat $S$, Homaee M (2015). Modeling sorghum response to irrigation water salinity at early growth stage. Agricultural Water Management 152:119-124. https://doi.org/10.1016/j.agwat.2015.01.008

Samreen T, Shah HU, Ullah S, Javid M (2017). Zinc effect on growth rate, chlorophyll, protein and mineral contents of hydroponically grown mungbeans plant (Vigna radiata). Arabian Journal of Chemistry 10:S1802-S1807. https://doi.org/10.1016/j.arabjc.2013.07.005

Shahverdi MA, Omidi H, Tabatabaei SJ (2019). Stevia (Stevia rebaudiana Bertoni) responses to NaCl stress: Growth, photosynthetic pigments, diterpene glycosides and ion content in root and shoot. Journal of the Saudi Society of Agricultural Sciences 18:355-360. 
Shaw B, Thomas T, Cooke D (2002). Responses of sugar beet (Beta vulgaris L.) to drought and nutrient deficiency stress. Plant Growth Regulation 37:77-83. https://doi.org/10.1023/A:1020381513976

Skorupa M, Gołębiewski M, Kurnik K, Niedojadło J, Kęsy J, Klamkowski K, Wójcik K, Treder W, Tretyn A, Tyburski J (2019). Salt stress vs. salt shock-the case of sugar beet and its halophytic ancestor. BMC Plant Biology 19:57. https://doi.org/10.1186/s12870-019-1661-X

Subramanyam K, Du Laing G, Van Damme EJ (2019). Sodium selenate treatment using a combination of seed priming and foliar spray alleviates salinity stress in rice. Frontiers in Plant Science 10:116. https://doi.org/10.3389/fpls.2019.00116

Talebi R, Ensafi M H, Baghebani N, Karami E, Mohammadi K (2013). Physiological responses of chickpea (Cicer arietinum) genotypes to drought stress. Environmental and Experimental Biology 11:9-15.

Wang K, Zhang L, Gao M, Lv L, Zhao Y, Zhang L, Li B, Han M, Alva AK (2013). Influence of salt stress on growth and antioxidant responses of two malus species at callus and plantlet stages. Pakistan Journal of Botany 45:375-381.

Yang Y, Guo Y (2018). Unraveling salt stress signaling in plants. Journal of Integrative Plant Biology 60:796-804. https://doi.org/10.1111/jipb.12689

Yemm E, Folkes B (1953). The amino acids of cytoplasmic and chloroplastic proteins of barley. Biochemical Journal 55:700-707. https://doi.org/10.1042/bj0550700

Zhang J, Jia W, Yang J, Ismail A M (2006). Role of ABA in integrating plant responses to drought and salt stresses. Field Crops Research 97:111-119. https://doi.org/10.1016/j.fcr.2005.08.018

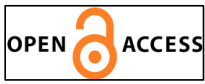

(c) (1)
The journal offers free, immediate, and unrestricted access to peer-reviewed research and scholarly work. Users are allowed to read, download, copy, distribute, print, search, or link to the full texts of the articles, or use them for any other lawful purpose, without asking prior permission from the publisher or the author.

License - Articles published in Notulae Botanicae Horti Agrobotanici Cluj-Napoca are Open-Access, distributed under the terms and conditions of the Creative Commons Attribution (CC BY 4.0) License. (C) Articles by the authors; UASVM, Cluj-Napoca, Romania. The journal allows the author(s) to hold the copyright/to retain publishing rights without restriction. 